\title{
INVARIANCE OF THE APPROXIMATELY REACHABLE SET UNDER NONLINEAR PERTURBATIONS *
}

\author{
Koichiro Naito $^{\dagger} \quad$ Thomas I. Seidman ${ }^{\ddagger}$
}

May 27, 2009

\begin{abstract}
This paper considers nonlinear perturbations of control systems with linear dynamics and seeks to analyze whether the approximately reachable set may be left unchanged by this perturbation. Under suitable conditions it is shown that this analysis may be reduced to the presumably simpler analysis of such invariance for a family of affine perturbations. Interest centers on the context of infinite-dimensional state spaces so the system may, for example, correspond to a hyperbolic or parabolic partial differential equation.
\end{abstract}

Keywords: approximate reachability, control, distributed parameter system, nonlinear, perturbation, invariance

\section{Introduction}

Reachability results for distributed parameter systems are hard to come by since, when the state space $\mathcal{X}$ is infinite-dimensional, the "standard" approaches (typically based on such implicit assumptions as local compactness, etc.) may no longer be applicable. This is especially true for nonlinear systems in a setting

\footnotetext{
* Received by the editors December 29, 1986; accepted for publication (in revised form) March 17, 1990.

${ }^{\dagger}$ Department of Economics, Senshuu University, Higashimita, Tama-ku, Kawasaki-shi, 152 Japan (e-mail: knaito@ponta.sucis.senshu-u.ac.jp).

${ }^{\ddagger}$ Department of Mathematics and Statistics, University of Maryland Baltimore County, Baltimore, Maryland 21228 (e-mail: seidman@umbc.bitnet, @umbc1.umbc.edu). This research has been partially supported by the United States Air Force Office of Scientific Research under grants AFOSR-82-0271 and AFOSR-87-0350 and by the National Science Foundation under grants CDR 85-00108 and ECS-8814788. Acknowledgment is also due to the System Research Center (University of Maryland) for its hospitality.
} 
for which the information available relates only to the approximately reachable sets for related linear problems. Our concern here is with the extension to this more general context of a mode of analysis treated in a sequence of earlier papers [11], [12], [15], [16].

We consider a nonlinear control system given by

$$
\dot{x}=\mathbf{A} x+\mathbf{F} x+\mathbf{B} u, \quad u \in \mathbb{U}_{a d}
$$

with $\mathbf{A}$ linear and $\mathbf{F}$ a nonlinear operator satisfying some suitable growth condition. The control operator $\mathbf{B}$ need not be linear and we may occasionally write $\mathbf{B}(u)$ to emphasize this; we set $\mathbb{W}_{a d}:=\left\{w=\mathbf{B}(u): u \in \mathbb{U}_{a d}\right\}$.

We wish to consider (1.1) as a "perturbation" of the control problem

$$
\dot{z}=\mathbf{A} z+\mathbf{B} u, \quad u \in \mathbb{U}_{a d}
$$

omitting the nonlinearity $\mathbf{F}$. For the moment we require only that (1.2) should have a meaningful solution for each $u \in \mathbb{U}_{a d}$ and we will consistently denote this solution by $z_{u}$. Setting

$$
\mathcal{Z}_{a d}:=\left\{z_{u} \text { satisfying (1.2) for some } u \in \boldsymbol{U}_{a d}\right\},
$$

part of our interpretation of "meaningful" is that $\mathcal{Z}_{a d}$ is to be considered in some ${ }^{1}$ Banach space $\boldsymbol{X}$ of functions on $[0, T]$ with continuity to $\mathcal{X}$ for the evaluation map $\mathbf{E}: z \mapsto z(T): \mathbb{X} \rightarrow \mathcal{X}$ so that we can speak about the reachable set

$$
\mathcal{K}_{0}:=\mathbf{E} \mathcal{Z}_{a d}:=\left\{\mathbf{E} z: z \in \mathcal{Z}_{a d}\right\}
$$

as a subset of the state space $\mathcal{X}$.

In considering (1.1), we observe that: for any particular solution $x=x_{u}$, the nonlinearity gives $\mathbf{F} x$ as some specific function $g$. This solution is then also a solution of an alternate perturbation of the system (1.2):

$$
\dot{x}=\mathbf{A} x+g+\mathbf{B} u, \quad u \in \mathbb{U}_{a d}
$$

using the same control $u$. We will consider the family of affine perturbations (1.4) with $g$ taken from some given subset $\mathcal{G}$ of a function space $\boldsymbol{V}$. The relation, of course, is that $\mathcal{G}$ is to contain each $\mathbf{F}[x(\cdot)]$ as $u$ ranges over $\mathbb{U}_{a d}$ in (1.1). Presumably the reachability analysis for (each of) the affinely perturbed problems (1.4) should be simpler than that for the original nonlinearly perturbed problem (1.1). For (1.4), (1.1) we will again be considering solutions in the same space $\mathscr{X}$ of $\mathcal{X}$-valued functions as we use for the "unperturbed" equation (1.2).

\footnotetext{
${ }^{1}$ In general we take $\boldsymbol{X}$ to be $C([0, T] \rightarrow \mathcal{X})$, which certainly ensures that the evaluation map $\mathbf{E}$ will be continuous. We do note that lesser regularity could be permitted away from the terminal time - in particular, to allow for the effect of "rough" initial data.
} 
It will be important for us to consider the relation of (1.1) to (1.2) from the viewpoint of (1.4). Given a solution $z=z_{u}$ for (1.2), we may write (1.1) as an equation for $y=x-z$ :

$$
\mathbf{G}: z \mapsto \mathbf{F}(y+z)=: g \text { such that } \dot{y}=\mathbf{A} y+g .
$$

For the moment this specification of a "map" $\mathbf{G}=\mathbf{G}_{\mathbf{F}}$ is purely formal but, of course, we will eventually want to have $\mathbf{G}: \mathscr{Z}_{a d} \rightarrow \mathcal{G}$.

The question we wish to consider is:

(Q) When must a state $\xi$ which is approximately reachable for (1.2) also be approximately reachable for the nonlinear system (1.1)?

Letting $\mathcal{K}_{\mathbf{F}} \subset \mathcal{X}$ denote the reachable set for (1.1) - i.e., the set of values for $\xi=x(T)$ as $x$ ranges over solutions of (1.1) with $u \in \mathbb{U}_{a d}$ - this asks whether $\mathcal{K}_{0} \subset \overline{\mathcal{K}}_{\mathbf{F}}$; we note that the complementary inclusion is, in general, much easier to analyze. More precisely, we are asking:

When can the question $(\mathbf{Q})$ above be analyzed (answered affirmatively) by considering the family of (presumably simpler) related questions for the affine problems (1.4) as $g$ ranges over $\mathcal{G}$ ?

In this form it should be clear that our concern is with the validation of $a$ mode of analysis. We are, for example, seeking conditions on (1.4) for $g \in$ $\mathcal{G}$ ensuring that $\overline{\mathcal{K}}$ is invariant under the perturbation: $(1.2) \longmapsto(1.1)$. The essential requirement will be a certain uniformity (over suitable bounded subsets of $\mathcal{G}$ ) for a measure of the approximate reachability; see (3.23) et seq.

We have already investigated the corresponding invariance of the (exactly) reachable set in a sequence of papers [11], [12], [15], [16]. The work presented here represents an extension of this work in two directions: the consideration of approximate rather than exact reachability and the consideration of control sets which do not form a linear space with linear $\mathbf{B}$.

We further note that our present approach to $(\mathbf{Q})$ is "one $\xi$ at a time" and so, with minor modification, lends itself to an analysis of approximate controllability for (1.1) in a less "global" context than the invariance of the title. The arguments used here put this work in the setting of "the fixed point approach to controllability" and we refer, e.g., to [5] and its references for further historical discussion of this approach.

\section{Some examples}

In this section, before beginning the analysis of reachability, we wish to provide some representative examples for which one can verify certain general hypotheses, $\left(\mathbf{H}_{1}\right)$ below, as to the settings to which our analysis applies. As previously noted, our concern is with settings for which the state space $\mathcal{X}$ is 
infinite-dimensional so that (1.2) may itself be an abstract formulation of a partial differential equation. We emphasize that our hypotheses do not restrict consideration to parabolic equations (compact, analytic semigroups). The analysis here could, in principle, be applied also to hyperbolic systems - although one expects that considerations of approximate reachability (compare [15], [17]) might then only be of interest if $\left\{\mathbf{B}(u): u \in \mathbb{U}_{a d}\right\}$ is not itself a linear space.

We turn now to some specific examples of systems governed by partial differential equations whose dynamics will satisfy the general hypotheses $\left(\mathbf{H}_{1}\right)$ below — but only indicate briefly the verifications. In $\S 6$ we will provide, following [15], some results which are sufficient to provide such verification for more general classes of problems. For each of these examples the spatial domain will be a bounded region $\Omega \subset \mathbb{R}^{m}$ with "smooth enough" boundary $\partial \Omega$; we set $\mathcal{Q}:=(0, T) \times \Omega$ and $\Sigma:=(0, T) \times \partial \Omega$.

Example 1. Our first example already exhibits many of our concerns for application. Consider boundary control of the nonlinear heat equation:

$$
\dot{x}=\Delta x+\varphi(\cdot, x, \nabla x) \text { on } \mathcal{Q}, \quad x_{n}=e^{u} \text { on } \Sigma
$$

as (1.1). The corresponding "linear" control problem is then

$$
\dot{z}=\Delta z \text { on } \mathcal{Q}, \quad z_{n}=w \text { on } \Sigma
$$

where $w:=e^{u}$. (Note that $x_{n}, z_{n}$ denote normal derivatives at $\partial \Omega$.)

We wish to take $\mathcal{X}:=L^{2}(\Omega)$ as state space. Note that the function $f(\xi):=$ $\varphi(\cdot, \xi(\cdot),[\nabla \xi](\cdot))$ is not well defined for general $\xi \in \mathcal{X}$ since the argument $\nabla \xi$ would then only be in $H^{-1}(\Omega)$ and so need not be a function at all for pointwise composition with $\varphi$. This is a situation for which the considerations of Lemma 3 below are important. If we assume that the scalar function $\varphi$ is (uniformly) Lipschitzian in its variables $\xi, \nabla \xi$, then we can introduce the auxiliary space $\mathcal{Y}:=L^{2}\left([0, T] \rightarrow H^{1}(\Omega)\right)$ and have Lipschitz continuity from $\boldsymbol{Y}$ to $\mathcal{V}:=L^{2}(\mathcal{Q})$ for the Nemytsky operator $\mathbf{F}: x \mapsto \varphi(\cdot, x, \nabla x)$. Fairly standard methods show that $\mathbf{L}$ is well defined and continuous from this $\boldsymbol{\nu}$ to $\boldsymbol{X}$ and to $\boldsymbol{y}$. Further, if one uses a suitable weighting (exponential in $t$ ) for the norms of $\boldsymbol{y}$ and $\boldsymbol{\nu}$, then one can arrange that $\vartheta<1$ so Lemma 3 will apply (provided we can take $\mathcal{Z} \hookrightarrow \boldsymbol{X} \cap \mathcal{Y})$.

Note that these considerations do not yet involve $\boldsymbol{U}_{a d}$ or $\mathcal{Z}_{a d}$ as $\mathbf{L}$ relates only to the problem with homogeneous boundary conditions (and, of course, homogeneous initial conditions).

Suppose we would wish to consider $\boldsymbol{U}_{a d}=\left\{u \in L^{2}(\Sigma): u>0\right.$ ae $\}$. Clearly we must begin by restricting this further, to those $u$ (and corresponding $w=e^{u}$ ) for which (2.7) and (2.6) have solutions in some satisfactory sense. If we take $w \in \mathbb{W}:=L^{2}(\Sigma)$, then it is standard that the solution map $\mathbf{S}$ defined by (2.7) is continuous and, indeed, compact from $\mathbb{W}$ to $\mathfrak{X} \cap \boldsymbol{V}$. One can then take, e.g., $\mathcal{Z}:=\mathbf{S W N}$, normed so that $\mathbf{S}$ is an isometry. By suitable density arguments one can show that the $\boldsymbol{X}$-closures of the sets of solutions for (2.6), (2.7), and the 
corresponding equation affinely perturbed by arbitrary $g \in \mathcal{V}$ will be the same whether one works with $\mathbb{W}_{a d}:=\{w \in \mathbb{W}: w \geq 1$ ae $\}$ or with the original $\boldsymbol{U}_{a d}$, restricted to $u$ giving solutions in $\mathscr{X}$. Thus one has equivalence in the sense of (3.17), below. This, together with Lemma 3 , gives $\left(\mathbf{H}_{1}\right)$. We note also that this $\mathcal{Z}_{a d}$ is convex and closed in $\mathcal{Z}$ since we obviously have $\mathbb{W}_{a d}$ convex and closed in NW.

Example 2. We next consider distributed control of the equation

$$
\dot{x}=\Delta x+\varphi(\cdot, x, \nabla x)+w \text { on } \mathcal{Q}, \quad x=0 \text { on } \Sigma,
$$

assuming that $\varphi: \mathcal{Q} \times \mathbb{R}^{m+1} \rightarrow \mathbb{R}$ is a "nice" function (i.e., with uniform bounds on enough derivatives). Again, (2.8) can be put into the abstract form (3.15) by taking the state space $\mathcal{X}$ to be $\mathcal{X}_{0}=L^{2}(\Omega)$ and then introducing $\mathbf{A}$ as the Laplacian on $\Omega$ with domain $\mathcal{D}(\mathbf{A}):=\left\{\xi \in H^{2}(\Omega) \subset \mathcal{X}_{0}:\left.\xi\right|_{\partial \Omega}=0\right\}$. Since $-\mathbf{A}$ is self-adjoint and positive, there is no difficulty (e.g., through the rate of decay of the eigenfunction expansion coefficients) in defining $[-\mathbf{A}]^{r}$ for all $r \geq 0$ and we set $\mathcal{X}_{\mu}:=\mathcal{D}\left([-\mathbf{A}]^{\mu / 2}\right)$; it is known [7], [8] that $\mathcal{X}_{\mu}=H^{\mu}(\Omega)$ for $0 \leq \mu<\frac{1}{2}$, that $\mathcal{X}_{\mu}=\left\{\xi \in H^{\mu}(\Omega):\left.\xi\right|_{\partial \Omega}=0\right\}$ for $\frac{1}{2}<\mu<\frac{5}{2}$, etc.

Consider controls $w \in \mathbb{W}=\mathbb{W}_{m}:=L^{2}\left([0, T] \rightarrow \mathcal{X}_{m}\right)$ for (fixed) $m \geq 0$. A standard semigroup estimate ${ }^{2}$ gives continuity for $\mathbf{L}_{[t]}: w \mapsto x(t)$ from $\mathbb{W}_{m}$ to $\mathcal{X}_{\mu}$ for each $\mu<m+1$ and a convolution estimate shows that $\mathbf{L} w=: x(\cdot)$ will be in $L^{2}\left([0, T] \rightarrow \mathcal{X}_{\mu}\right)$ for $\mu<m+2$. We get

$$
x(\cdot) \in \mathfrak{X}_{\mu}:=C\left([0, T] \rightarrow \mathcal{X}_{\mu}\right) \cap L^{2}\left([0, T] \rightarrow \mathcal{X}_{\mu+1}\right)
$$

for each $\mu<m+1$. On the other hand, for any $\xi \in \mathcal{X}_{m+2}$ we can set $w_{*}(t):=(\xi-t \mathbf{A} \xi) / T$ to get $w_{*} \in \mathbb{W}_{m}$ and $\mathbf{L}_{[t]} w_{*}=(t / T) \xi$. Hence, $\mathcal{X}_{m+2} \subset$ $\mathcal{K}_{0}\left(\mathbb{W}_{m}\right) \subset \cap_{\mu<m+1} \mathcal{X}_{m}$. Any more precise estimate of $\mathcal{K}_{0}\left(\mathbb{W}_{m}\right)$ would be much more difficult to obtain - even in this "simple" setting.

In considering the nonlinear partial differential equation (2.8), our assumptions make $\varphi$ uniformly Lipschitzian in $x, \nabla x$, so a standard (Picard iteration) argument gives existence of a (unique) solution of the nonlinear equation (2.8) in $\mathbb{X}_{0}$ for, say, any control $w \in \mathbb{W}_{0}=L^{2}(\mathcal{Q})$; compare the proof of Lemma 3 below and the proof of Lemma 4 in [15]. This then gives $\varphi(\cdot, x, \nabla x)=: \mathbf{F} x=$ : $\mathbf{G}_{\mathbf{F}} w=: g$ again in $L^{2}(\mathcal{Q})$ so $[g+w] \in \mathbb{W}_{0}$ and by the analysis above, we then have $x \in \boldsymbol{X}_{\mu}$ for arbitrary $\mu<1$ and this gives $\mathcal{K}_{\mathbf{F}} \subset \cap_{\mu<1} \mathcal{X}_{\mu}$. For smoother controls one can similarly obtain the regularity result: $\mathcal{K}_{\mathbf{F}}\left(\mathbb{W}_{m}\right) \subset \cap_{\mu<m+1} \mathcal{X}_{\mu}$ provided one has an estimate of the form:

$$
\left\|(-\mathbf{A})^{\mu / 2} \mathbf{F} x\right\| \leq C\left[1+\left\|(-\mathbf{A})^{(\mu+1) / 2} x\right\|\right]
$$

\footnotetext{
${ }^{2}$ Since $\mathbf{A}$ generates an analytic semigroup on $\mathcal{X}_{0}$ one has

$$
\left\|(-\mathbf{A})^{r} \mathbf{S}(\tau)\right\| \leq C \tau^{-r}
$$

on bounded intervals for $r \geq 0$ and with $C$ depending on $r$ and the interval.
} 
for such $\mu$. The estimate (2.10) follows from the assumed regularity of $\varphi$ while $\mu<\frac{1}{2}$ but the boundary conditions then intervene; one will only continue to have (2.10), at least while $\mu<\frac{5}{2}$, provided that, in addition to the bounds on derivatives for $\varphi$, one were to require that $\varphi(\cdot, 0, \cdot)=0$ on $\partial \Omega$ so $\left.x\right|_{\partial \Omega}=0$ also implies $\left.[\mathbf{F} x]\right|_{\partial \Omega}=0$.

For the nonlinear equation (2.8) there is no longer a simple explicit calculation to give a useful lower bound for $\mathcal{K}_{\mathbf{F}}\left(\mathbb{W}_{m}\right)$. Taking $\mathcal{X}=\mathcal{X}_{0}$, for example, we have $\mathcal{X}_{m+2}$ dense in $\mathcal{X}$ for arbitrary $m$ so $\overline{\mathcal{K}}_{0}\left(\mathbb{W}_{m}\right)=\mathcal{X}$ and we would similarly like to show that $\overline{\mathcal{K}}_{\mathbf{F}}\left(\mathbb{W}_{m}\right)=\mathcal{X}$. Under the assumptions we are now imposing, we can consider, e.g., $1<m<\frac{3}{2}$ and get

$$
\mathbf{L}_{[T]} g \in \mathcal{D}(\mathbf{A})=\left[\mathcal{D}\left([-\mathbf{A}]^{m+1}\right), \mathcal{X}\right]_{\vartheta} \subset\left[\mathcal{K}_{0}\left(\mathbb{W}_{m}, \mathcal{X}\right]_{\vartheta}\right.
$$

with $\vartheta=m /(m+1)$ as parameter for the interpolation spaces, corresponding to (5.41). Thus, looking ahead, we note that the assumptions of Theorem 5 will then be satisfied if one further imposes a growth condition on $\varphi$ to strengthen (2.10) so as to get $(6.48)$ with $r<1 /(m+1)$.

Example 3. For our next example we take $\Omega=(0,1) \subset \mathbb{R}^{1}$ and consider

$$
\begin{array}{ccl}
\dot{x}=x^{\prime \prime}+\varphi\left(x, x^{\prime}\right) & \text { on } & \mathcal{Q}:=(0, T) \times(0,1), \\
x(\cdot, 0)=u(\cdot), x(\cdot, 1)=0 & \text { on } & (0, T) .
\end{array}
$$

As above we assume $\varphi$ is uniformly Lipschitzian and take $\mathcal{X}:=L^{2}(0,1)$; we now wish to take

$$
\mathbb{W}_{a d}=\mathbb{U}_{a d}:=\{\text { integer-valued step functions on }(0, T)\} \text {. }
$$

We again take $\mathcal{Y}:=L^{2}\left([0, T] \rightarrow H^{1}(0,1)\right)$ and $\mathcal{V}:=L^{2}(\mathcal{Q})$. Since we can "restart" the equations at each of the (finitely many) jumps of $u \in \boldsymbol{U}_{a d}$, there is no difficulty with the solvability, for each such $u$, of the various equations: solutions will be piecewise smooth. Our difficulty is now with the continuity of the maps and with the desired convexity of $\mathbb{Z}_{a d}$.

Suppose the scalar function $\varphi$ also satisfies a growth condition $\varphi(r, s) \leq$ $a_{0}+a_{1}|r|$ so the Nemytsky operator $\mathbf{F}$ satisfies $|[\mathbf{F} x](t)|_{\mathcal{X}} \leq C_{0}+C_{1}|x(t)|_{\mathcal{X}}$, independent of $x^{\prime}$. In this case, a bound on $u$ in, say, $L^{2}(0, T)$ gives solutions, for (2.11) and for the corresponding linear equations, in a compact subset of $\mathbb{X}$ and, using interior regularity, such that the corresponding gradients are in a compact subset of, say, $L^{2}((0, T) \times(a, b))$ for any $0<a<b<1$. It follows, extracting suitable convergent subsequences for which the gradients are converging pointwise ae, that if one considers any bounded set in $L^{2}(0, T)$ then all limits of the solutions will again be solutions in $\mathfrak{X}$. We now observe (compare Lemma 5) that the set $\mathbb{U}_{a d}$ above and the set $\mathbb{W}:=H_{0}^{1}(0, T)$ have the same (sequential) weak closure in $L^{2}(0, T)$, i.e., all of $L^{2}(0, T)$, so the $\boldsymbol{X}$-closures of the sets of solutions (and so the corresponding approximately reachable sets) will be the same for each of these as for $L^{2}(0, T)$ - restricted to those $u$ for which there 
are, indeed, solutions in $\boldsymbol{X}$ satisfying the boundary conditions in a meaningful sense. It follows that we can take $\mathbb{W}_{a d}^{\prime}:=\mathbb{W}$ and $\mathcal{Z}_{a d}^{\prime}=\mathcal{Z}:=\mathbf{S} \mathbb{W}_{a d}^{\prime}$ (noting that we do have continuity and compactness for $\mathbf{S}: \mathbb{W} \rightarrow \boldsymbol{X} \cap \boldsymbol{y}$ ) with equivalence in the sense of (3.17). We will have $\left(\mathbf{H}_{1}\right)$ and the needed convexity if we work with this $\mathcal{Z}_{a d}$.

Example 4. For our final example we consider a quasi-linear wave equation

$$
\ddot{x}=\Delta x+\varphi(\cdot, x) \text { on } \mathcal{Q},\left.\quad x\right|_{\Sigma}=u
$$

where we assume $\varphi$ is smooth, uniformly Lipschitzian and with a growth condition $|\varphi| \leq C(1+|x|)^{\bar{r}}$ with $\bar{r}<1$. We assume homogeneous initial conditions: $x(0)=0=\dot{x}(0)$ and that the controls $u \in \mathbb{U}_{a d}$ are to be taken smooth enough to have an extension to $\mathcal{Q}$, again denoted by $u$, with, say, $q:=[\ddot{u}-\Delta u] \in L^{2}(\mathcal{Q})$. This will ensure that the solution $z=z_{u}$ of the corresponding form of (1.2)

$$
\ddot{z}=\Delta z \text { on } \mathcal{Q},\left.\quad z\right|_{\Sigma}=u
$$

will be in $\mathcal{Z}:=C^{1}\left([0, T] \rightarrow L^{2}(\Omega) \cap C\left([0, T] \rightarrow H^{1}(\Omega)\right)\right.$, using an estimate obtained by multiplying (2.13) by $[z-u]$, integrating, and applying the Gronwall Inequality. If we take $\boldsymbol{V}:=L^{2}(\mathcal{Q})$, then a similar estimate shows the continuity of $\mathbf{L}: \boldsymbol{V} \rightarrow \mathcal{Z}$. Finally, we note that (1.5) becomes

$$
\ddot{y}=\Delta y+\varphi(\cdot, y+z) \text { on } \mathcal{Q},\left.\quad y\right|_{\Sigma}=0
$$

and a standard contraction mapping argument gives existence of a solution $y \in \mathcal{Z}$, using the Lipschitz property of $\varphi$, with an estimate $|y|=\mathcal{O}\left(|z|^{\bar{r}}\right.$ ) where $|y|$ is the $\mathcal{Z}$-norm and $|z|$ is the $\boldsymbol{V}$-norm. The compactness of the embedding $\mathcal{Z} \hookrightarrow \boldsymbol{V}$ then completes the verification of the general hypotheses $\left(\mathbf{H}_{1}\right)$ with the growth condition (4.28).

We note two particular cases of interest from the viewpoint of reachability: (1) in the one-dimensional case with $\boldsymbol{U}_{a d}$ consisting of functions vanishing at one end of $\Omega$ and nonnegative at the other, it is known [13] that $\mathcal{K}_{0}$ is dense in, say, $\mathcal{X}=L^{2}(\Omega)$ for $T$ large enough; (2) for $\Omega \subset \mathbb{R}^{m}$ with $u \in \mathbb{U}_{\text {ad }}$ having support in some small fixed subset $\Gamma \subset \partial \Omega$ and/or with $T$ not too large, then the reachable set will only be some (small) part of $\mathcal{X}$ and it is interesting to ask whether a geometric restriction on the support in $\mathcal{Q}$ for $\varphi$ could provide the hypotheses for invariance.

\section{Formulation and notation}

We have already introduced the set $\mathbb{Z}_{a d}$ of solutions of (1.2) (in some fixed sense) as the control $u$ ranges over $\boldsymbol{U}_{a d}$ and the (formal) operator $\mathbf{E}: x \mapsto x(T)$. We now also introduce the linear solution operator $\mathbf{L}$ :

$$
\mathbf{L}: v \mapsto x \text { such that } \dot{x}=\mathbf{A} x+v
$$


(with homogeneous initial conditions) for suitable $v(\cdot)$. Note that (1.2) and (1.4) have linear dynamics (i.e., linear in $z$ or $x$ although not necessarily in $u$ ) while the dynamics given by the perturbed equation (1.1) are quasi-linear.

We have already made our first basic observation: neither $\mathbf{B}$ nor $u \in \boldsymbol{U}_{\text {ad }}$ (nor their individual properties) can be relevant to any of (1.2), (1.1), (1.4), but only $w:=\mathbf{B}(u)$, considered as an element of some space $\mathbb{W}$. Indeed, we have seen that the only really relevant entity is $z=z_{u}$, the corresponding solution of (1.2). Thus, the effects of control are entirely determined by the set $\mathcal{Z}_{a d}$.

Expressed in terms of this (formal) operator $\mathbf{L}$, the differential equations (1.1) and (1.4) then take the abstract forms:

$$
\begin{array}{ll}
x=\mathbf{L F} x+z & \left(z \in \mathcal{Z}_{a d}\right), \\
x=\mathbf{L} g+z & \left(z \in \mathcal{Z}_{a d}, g \in \mathcal{G}\right),
\end{array}
$$

where $\mathcal{Z}_{\text {ad }}$ is now simply a (specified) subset of some function space $\mathcal{Z}$ and $\mathcal{G}$ is a specified subset of another function space $\boldsymbol{V}$. Until one specifies the spaces involved this is purely formal but we note here that, although we refer for convenience to (1.1), (1.2), (1.4), we will always be interpreting "solution" in the present sense: through the abstract operator equations (3.15), (3.16) with any hypotheses and interpretations to be attached to these. Except for this section, the (motivating) earlier examples, and the final section, our considerations are independent of any interpretation of (3.15) and (3.16) as differential equations.

Note that, since we consider the equations (1.2), etc., with fixed initial conditions, it is always possible, with no loss of generality, to translate the problem by some fixed trajectory $z_{0}$, correspondingly modifying $\mathbf{F}, \mathbb{Z}_{a d}$, and all reachable sets. Henceforth, for expository simplicity, we do assume that, ab initio, the problem has been formulated with homogeneous initial conditions so that one has $z_{u}=\mathbf{L} w$ where $w=\mathbf{B}(u)$. At the same time, once one has avoided consideration of any (essentially irrelevant) problems with regularity near the initial time, it is convenient to assume that all our solutions are elements of the fixed space $\mathcal{X}:=C([0, T] \rightarrow \mathcal{X})$ so that, in particular, the operator $\mathbf{E}: \mathcal{X} \rightarrow \mathcal{X}$ is always well defined and continuous. We also assume that $\mathcal{Z}_{a d} \subset \boldsymbol{X}$ settheoretically, but may find it convenient to topologize it somewhat differently: as $\mathcal{Z}_{\text {ad }} \subset \mathcal{Z}$; e.g., if there is a continuous embedding $\mathcal{Z} \hookrightarrow \boldsymbol{X}$.

Our underlying set of "solvability hypotheses" is:

$\left(\mathbf{H}_{1}\right)$ Let each of the following hold:

(i) Equation (3.14) has a (unique) solution $x=\mathbf{L} v \in \mathbb{X}^{\text {for each }}$ $v \in \mathcal{V}$; the linear map $\mathbf{L}$ is well-defined and continuous from $\boldsymbol{V}$ to $\mathbb{X}$.

(ii) For each $z \in \mathbb{Z}_{a d}$ there is a unique solution $x$ of (3.15) and we assume that $g=\mathbf{F} x$ is in $\mathcal{V}$, i.e., there is a well-defined (nonlinear) map $\mathbf{G}=\mathbf{G}_{\mathbf{F}}: \mathcal{Z}_{a d} \rightarrow \mathcal{V}: z \mapsto g$ which we 
assume is continuous. By (i) we then have $x \in \mathbb{X}$ for the solution $x$ of (3.15).

(iii) The map $\mathbf{G}: \mathbb{Z}_{a d} \rightarrow \mathcal{V}$ is compact.

Compare (1.5) for the definition of the map G. In $\S 4$ we will consider some specific examples involving partial differential equations and some classes of settings for which these abstract hypotheses can be verified.

Having formulated the "dynamics" of the problem — introducing the relevant spaces and the operators $\mathbf{L}$ and $\mathbf{G}_{\mathbf{F}}$ to obtain (3.15), (3.16) - we next wish to consider the various reachable and approximately reachable sets. We now define $\mathbf{L}_{[t]}: v \mapsto[\mathbf{L} v](t)$ (so, in particular, $\mathbf{E L}=\mathbf{L}_{[T]}$ ) and set

$$
\mathbf{T}=\mathbf{T}_{\mathbf{F}}:=\mathbf{L}_{[T]} \mathbf{G}+\mathbf{E}: \mathcal{Z}_{a d} \rightarrow \mathcal{X}: z \mapsto \xi:=\mathbf{E} x \text { such that }(3.15)
$$

Clearly, in view of $\left(\mathbf{H}_{1}\right)$, the operator $\mathbf{T}$ is continuous. If, for arbitrary $\mathbb{Z}_{*} \subset \mathbb{Z}$, we define:

$$
\begin{aligned}
& \mathcal{K}_{\mathbf{F}}\left(\mathbb{Z}_{*}\right):=\left\{\mathbf{E} x:(3.15) \text { for } z \in \mathbb{Z}_{*}\right\}=\left\{\mathbf{T}_{\mathbf{F}} z: z \in \mathbb{Z}_{*}\right\}, \\
& \mathcal{K}_{0}\left(\mathbb{Z}_{*}\right):=\mathbf{E} \mathbb{Z}_{*}:=\left\{\mathbf{E} z: z \in \mathbb{Z}_{*}\right\} \text {, } \\
& \mathcal{K}_{g}\left(\mathbb{Z}_{*}\right):=\left\{\left(\mathbf{L}_{[T]} g+\mathbf{E} z\right): z \in \mathbb{Z}_{*}\right\}=\mathbf{L}_{[T]} g+\mathcal{K}_{0}\left(\mathbb{Z}_{*}\right),
\end{aligned}
$$

then the (exactly) reachable sets for (1.2), (1.1), and (1.4) will be $\mathcal{K}_{0}=\mathcal{K}_{0}\left(\mathbb{Z}_{\text {ad }}\right)$, $\mathcal{K}_{\mathbf{F}}=\mathcal{K}_{\mathbf{F}}\left(\mathcal{Z}_{a d}\right)$, and $\mathcal{K}_{g}=\mathcal{K}_{g}\left(\mathcal{Z}_{a d}\right)$, respectively. The corresponding approximately reachable sets are then the $\mathcal{X}$-closures: $\overline{\mathcal{K}}_{0}, \overline{\mathcal{K}}_{\mathbf{F}}, \overline{\mathcal{K}}_{g}$, respectively (i.e., $\overline{\mathcal{K}_{0}\left(\mathcal{Z}_{a d}\right)}$, etc. $)$. We make here our second basic observation: for present purposes we may always replace the "original" $\mathbb{Z}_{a d}$ at our convenience with any other set $\mathbb{Z}_{a d}^{\prime}$ for which the approximately reachable sets are the same:

$$
\overline{\mathcal{K}}_{0}\left(\mathcal{Z}_{a d}^{\prime}\right)=\overline{\mathcal{K}}_{0}\left(\mathbb{Z}_{a d}\right), \overline{\mathcal{K}}_{g}\left(\mathcal{Z}_{a d}^{\prime}\right)=\overline{\mathcal{K}}_{g}\left(\mathbb{Z}_{a d}\right), \overline{\mathcal{K}}_{\mathbf{F}}\left(\mathcal{Z}_{a d}^{\prime}\right)=\overline{\mathcal{K}}_{\mathbf{F}}\left(\mathcal{Z}_{a d}\right)
$$

In particular, the continuity of $\mathbf{G}$ assumed in $\left(\mathbf{H}_{1}\right)$ (ii) will mean that we can always take $\mathscr{Z}_{a d}$ closed, i.e., replacing it by its closure with no loss of generality. As part of this observation we also note that $\mathcal{Z}_{a d}$ - or, equivalently, $\mathbb{Z}_{a d}^{\prime}$ satisfying (3.17) — is of purely set-theoretic significance so the relevant topology is at our convenience.

It is sometimes convenient to introduce explicitly the intermediate space WW such that $\mathbf{B}(u) \in \mathbb{W}_{a d} \subset \mathbb{W}$ so $\mathbb{Z}_{a d}=\mathbf{S} \mathbb{W}_{a d}$ and to consider the map: $u \mapsto w:=\mathbf{B}(u) \mapsto z=z_{u}$ defined by (1.2). The second "factor" of this is the (linear) solution map for (1.2):

$$
\mathbf{S}: \mathbb{W} \rightarrow \mathbb{Z}: w \mapsto z
$$

and we now write $\mathbb{W}_{a d} \equiv \mathbb{N W}_{a d}^{\prime}$ if $\mathbb{Z}_{a d}:=\mathbf{S} \mathbb{W}_{a d}$ and $\mathbb{Z}_{a d}^{\prime}:=\mathbf{S} \mathbb{W}_{a d}^{\prime}$ are equivalent in the sense of (3.17). 
In our previous work we assumed, with $\mathcal{G}_{*}=\mathcal{V}$, that the exactly reachable set for (1.2) was invariant under all the affine perturbations (1.4) for $g \in \mathcal{V}$, i.e., that

$$
\mathcal{K}_{g}=\mathcal{K}_{0} \text { for each } g \in \boldsymbol{G}_{*} .
$$

The natural reachability condition for us to consider in attempting a corresponding treatment of approximate controllability would seem to be

$$
\overline{\mathcal{K}}_{g}=\overline{\mathcal{K}}_{0} \text { for each } g \in \mathcal{G}_{*}
$$

but we will need to strengthen half of this, the inclusion

$$
\mathcal{K}_{0} \subset \overline{\mathcal{K}}_{g}
$$

to obtain the desired result. Suppose, given $\xi \in \mathcal{X}$ and $\varepsilon>0$, we introduce the set-valued function $\mathcal{C}(\cdot)=\mathcal{C}(\cdot ; \xi, \varepsilon)$ defined on $\mathcal{\nu}$ by

$$
\mathcal{C}(g ; \xi, \varepsilon):=\left\{z \in \mathbb{Z}_{a d}:\left|\xi-\left[\mathbf{L}_{[T]} g+\mathbf{E} z\right]\right|_{\mathcal{X}} \leq \varepsilon\right\}
$$

Then (3.21) just means that $\mathcal{C}(g ; \xi, \varepsilon)$ is nonempty for each $\xi \in \mathcal{K}_{0}$ and each $\varepsilon>0$. Fixing $\xi \in \mathcal{K}_{0}$ and $\varepsilon>0$, we now set

$$
\nu(g)=\nu(g ; \xi, \varepsilon):=\inf \left\{|z|_{\mathcal{Z}}: z \in \mathcal{C}(g ; \xi, \varepsilon)\right\}
$$

for $g \in \mathcal{V}$ and then, for $R>0$, define

$$
\beta(R)=\beta(R ; \xi, \varepsilon):=\sup \left\{\nu(\mathbf{G} z ; \xi, \varepsilon): z \in \mathbb{Z}_{a d},|z|_{Z^{Z}} \leq R\right\}
$$

To have each $\nu(g)$ finite (for $\varepsilon>0$ and $g \in \mathcal{G}_{*}$ ) just means that $\xi \in \overline{\mathcal{K}}_{g}$ (for each $\left.g \in \mathcal{G}_{*}\right)$ so $(3.21)$ means that $\nu(g ; \xi, \varepsilon)<\infty$ for each $\varepsilon>0$ and every $\xi \in \mathcal{K}_{0}$. This does not yet mean that $\beta$ will be finite and we will express the desired strengthening of (3.21) quantitatively in terms of $\nu(\cdot)$ and $\beta(\cdot)$.

Returning to the condition (3.20), we finish this section with some observations about the structure of the set $\boldsymbol{V}_{a}=\mathcal{V}_{a}\left(\mathbb{Z}_{a d}\right)$ given by

$$
\mathcal{V}_{a}:=\left\{g \in \mathcal{V}: \overline{\mathcal{K}}_{g}=\overline{\mathcal{K}}_{0}\right\}=\left\{g \in \mathcal{V}: \mathbf{L}_{[T]} g+\overline{\mathcal{K}}_{0}=\overline{\mathcal{K}}_{0}\right\}
$$

noting that (3.20) just asserts that $\mathcal{G}_{*} \subset \mathcal{V}_{a}$. Since we always assume $\mathbf{L}_{[T]}$ is continuous, we always have $\boldsymbol{V}_{a}$ closed in $\boldsymbol{V}$.

LemMA 1. $\mathcal{V}_{a}$ is closed under addition and subtraction. If $\overline{\mathcal{K}}_{0}$ is convex, then $\mathcal{V}_{a}$ is a (closed) subspace of $\mathcal{V}$.

Proof. Suppose $g, g^{\prime} \in \mathcal{V}_{a}$. We first wish to show that $\mathcal{K}_{\bar{g}} \subset \overline{\mathcal{K}}_{0}$ where $\bar{g}:=$ $g-g^{\prime}$, i.e., for any $\bar{\xi} \in \mathcal{K}_{\bar{g}}$ and $\varepsilon>0$ that there exists $\bar{z} \in \mathbb{Z}_{\text {ad }}$ with $|\bar{\xi}-\mathbf{E} \bar{z}| \leq \varepsilon$. To start, we have $\bar{\xi}=\mathbf{L}_{[T]} \bar{g}+\mathbf{E} z_{0}=\xi_{1}-\mathbf{L}_{[T]} g^{\prime}$ with $\xi_{1}:=\mathbf{L}_{[T]} g+\mathbf{E} z_{0}$. Since $\xi_{1} \in \mathcal{K}_{g}$ and $g \in \mathcal{V}_{a}$ gives $\mathcal{K}_{g} \subset \overline{\mathcal{K}}_{0}$, there must be $z_{1} \in \mathbb{Z}_{\text {ad }}$ such that $\left|\xi_{1}-\mathbf{E} z_{1}\right| \leq \varepsilon / 2$. Now $\mathbf{E} z_{1} \in \mathcal{K}_{0}$ and $g^{\prime} \in \mathcal{V}_{a}$ gives $\mathcal{K}_{0} \subset \overline{\mathcal{K}}_{g^{\prime}}=\mathbf{L}_{[T]} g^{\prime}+\overline{\mathcal{K}}_{0}$ so $\left(\mathbf{E} z_{1}-\mathbf{L}_{[T]} g^{\prime}\right) \in \overline{\mathcal{K}}_{0}$ and there must be $\bar{z} \in \mathbb{Z}_{a d}$ with $\left|\left[\mathbf{E} z_{1}-\mathbf{L}_{[T]} g^{\prime}\right]-\mathbf{E} \bar{z}\right| \leq \varepsilon / 2$. 
Since $\bar{\xi}-\mathbf{E} \bar{z}=\left(\xi_{1}-\mathbf{E} z_{1}\right)+\left(\mathbf{E} z_{1}-\left[\mathbf{L}_{[T]} g^{\prime}+\mathbf{E} \bar{z}\right]\right)$, this gives $|\xi-\mathbf{E} \bar{z}| \leq \varepsilon$ as desired so $\bar{\xi} \in \overline{\mathcal{K}}_{0}$. This shows $\mathbf{L}_{[T]} \bar{g}+\mathcal{K}_{0} \subset \overline{\mathcal{K}}_{0}$ for $\bar{g}=g-g^{\prime}$. Reversing the roles of $g, g^{\prime}$ gives $-\mathbf{L}_{[T]} \bar{g}+\mathcal{K}_{0} \subset \mathcal{K}_{0}$ or $\mathcal{K}_{0} \subset \overline{\mathcal{K}}_{\bar{g}}$. Combining gives $\overline{\mathcal{K}}_{0}=\overline{\mathcal{K}}_{\bar{g}}$ so $\bar{g} \in \boldsymbol{V}_{a}$ for $\bar{g}=g-g^{\prime} \in \boldsymbol{V}_{a}-\boldsymbol{V}_{a}$, i.e., $\boldsymbol{V}_{a}$ is closed under subtraction. Trivially, $0 \in \boldsymbol{V}_{a}$ so $g^{\prime} \in \boldsymbol{V}_{a}$ gives $-g^{\prime} \in \boldsymbol{V}_{a}$ whence $\bar{g}=g-\left(-g^{\prime}\right)=g+g^{\prime}$ is in $\boldsymbol{V}_{a}$ for $g, g^{\prime} \in \mathcal{V}_{a}$, i.e., $\boldsymbol{V}_{a}$ closed under addition also.

If we only show that convexity of $\overline{\mathcal{K}}_{0}$ implies that of $\mathcal{V}_{a}$, then the algebraic closure above shows that $\boldsymbol{\nu}_{a}$ is a subspace. Suppose, then, $\bar{g}$ is any convex combination of $\boldsymbol{V}_{a}$ so $\bar{g}=\sum c_{j} g_{j}$ with $c_{j}>0, \sum c_{j}=1, g_{j} \in \boldsymbol{V}_{a}$. For any $\xi \in \mathcal{K}_{0}$ we have $\mathbf{L}_{[T]} \bar{g}+\xi=\sum c_{j}\left(\mathbf{L}_{[T]} g_{j}+\xi\right)$. As each $g_{j} \in \boldsymbol{V}_{a}$ we have each $\left(\mathbf{L}_{[T]} g_{j}+\xi\right) \in \mathcal{\mathcal { K }}_{0}$ so convexity of $\overline{\mathcal{K}}_{0}$ gives $\left(\mathbf{L}_{[T]} \bar{g}+\xi\right) \in \overline{\mathcal{K}}_{0}$. This, for each $\xi \in \mathcal{K}_{0}$, gives $\mathcal{K}_{\bar{g}} \subset \overline{\mathcal{K}}_{0}$. By Lemma 1 we have also $-\bar{g}=\sum c_{j}\left(-g_{j}\right)$ a convex combination of $\boldsymbol{\nu}_{a}$ so $\left[\mathbf{L}_{[T]}(-\bar{g})+\xi\right] \in \overline{\mathcal{K}}_{0}$ for each $\xi \in \mathcal{K}_{0}$, i.e., $\xi \in\left[\mathbf{L}_{[T]} \bar{g}+\overline{\mathcal{K}}_{0}\right]=\overline{\mathcal{K}}_{\bar{g}}$. Combining gives $\overline{\mathcal{K}}_{0}=\overline{\mathcal{K}}_{\bar{g}}$ so $\bar{g} \in \boldsymbol{V}_{a}$.

Note that closure under addition shows that $\boldsymbol{V}_{a}$ is always unbounded (except for the trivial case: $\boldsymbol{\nu}_{a}=\{0\}$ ), so $\mathbb{Z}_{a d}$ must then also be unbounded. Note that $\overline{\mathcal{K}}_{0}$ will certainly be convex if $\mathcal{Z}_{a d}$ is convex.

\section{Reachability}

At this point we begin our analysis of approximate reachability by proving the trivial part of invariance: if, for each affine perturbation by $g \in \mathcal{G}_{*}:=\mathbf{G} \mathcal{Z}_{\text {ad }}$ in (1.4), one can reach no state which is not already approximately reachable for (1.2), then the nonlinear perturbation (1.1) can also produce no new reachable states.

Lemma 2. Assume $\left(\mathbf{H}_{1}\right)(\mathrm{ii})$ and the reachability inclusion

$$
\mathcal{K}_{g} \subset \overline{\mathcal{K}}_{0} \text { for each } g \in \mathcal{G}_{*},
$$

i.e., $\xi+\mathbf{L}_{[T]} g \in \overline{\mathcal{K}}_{0}$ for $\xi \in \mathcal{K}_{0}, g \in \mathcal{G}_{*}$. Then $\overline{\mathcal{K}}_{\mathbf{F}} \subset \overline{\mathcal{K}}_{0}$.

Proof. Since $\overline{\mathcal{K}}_{\mathbf{F}}=\cap_{\varepsilon>0}\left[\mathcal{K}_{\mathbf{F}}+\mathcal{B}_{\varepsilon}\right]$ with $\mathcal{B}_{\varepsilon}:=\{\xi \in \mathcal{X}:|\xi|<\varepsilon\}$, we have $\xi \in \overline{\mathcal{K}}_{\mathbf{F}}$ if and only if for each $\varepsilon>0$ there is some $\xi_{\varepsilon}=\mathbf{T} z_{\varepsilon} \in \mathcal{K}_{\mathbf{F}}$ with $\left|\xi_{\varepsilon}-\xi\right|<\varepsilon$. Setting $g_{\varepsilon}:=\mathbf{G} z_{\varepsilon}$, we then have $\mathbf{T} z_{\varepsilon}=\mathbf{L}_{[T]} g_{\varepsilon}+\mathbf{E} z_{\varepsilon}$ so $\xi_{\varepsilon} \in \mathcal{K}_{g_{\varepsilon}}$. This shows that we always have

$$
\overline{\mathcal{K}}_{\mathbf{F}} \subset \bigcap_{\varepsilon>0} \bigcup_{g \in \mathcal{G}_{*}}\left[\mathcal{K}_{g}+\mathcal{B}_{\varepsilon}\right]=\bigcap_{\varepsilon>0}\left[\left(\bigcup_{g \in \mathcal{G}_{*}} \mathcal{K}_{g}\right)+\mathcal{B}_{\varepsilon}\right] .
$$

Now, if one has (4.26), then the right side of (4.27) will be in $\cap_{\varepsilon>0}\left[\mathcal{K}_{0}+\mathcal{B}_{\varepsilon}\right]=\overline{\mathcal{K}}_{0}$ as asserted.

For comparison we note that our previous work [11], [12], [15], [16] obtained essentially the following result. 
THEOREM 1. Let $\mathcal{Z}_{\text {ad }}=\mathscr{Z}$ be a Banach space ${ }^{3}$ and assume $\left(\mathbf{H}_{1}\right)$ together with the growth condition

$$
\left|\mathbf{G}_{\mathbf{F}} z\right|_{\mathcal{\nu}} \leq C_{0}+C_{1}|z|_{\mathcal{Z}}^{\bar{r}}
$$

for some $\bar{r}<1$. Assume the reachability invariance $(3.19): \mathcal{K}_{g}=\mathcal{K}_{0}$ for each $g \in \mathcal{V}$. Then $\mathcal{K}_{\mathbf{F}}=\mathcal{K}_{0}$, i.e., the exactly reachable set is then invariant under the perturbation by $\mathbf{F}$.

(Of course this implies invariance of the approximately reachable set: we also have $\overline{\mathcal{K}}_{\mathbf{F}}=\overline{\mathcal{K}}_{0}$.)

Proof. We only sketch the proof here to fix the ideas; for details, see [15]. From the inclusion $\mathcal{K}_{g} \subset \mathcal{K}_{0}$, we can argue as in Lemma 2 to have $\mathcal{K}_{\mathbf{F}} \subset \mathcal{K}_{0}$. The principal effort must go into showing that one also has the reverse inclusion $\mathcal{K}_{0} \subset \mathcal{K}_{\mathbf{F}}$.

The proof begins by noting equivalence of (3.19) to the range condition

$$
\mathbf{L}_{[T]} \mathcal{V} \subset \mathbf{E} \mathcal{Z}
$$

Then the nullspace of the continuous linear map:

$$
[g,\{z\}] \mapsto \mathbf{L}_{[T]} g+\mathbf{E} z: \mathcal{V} \times[\mathcal{Z} / \mathcal{N}(\mathbf{E})] \rightarrow \mathcal{X}
$$

will be the graph of a linear operator: $g \mapsto\{z\}$ which is bounded by the Closed Graph Theorem. By the Michael Selection Theorem [10], there is a continuous

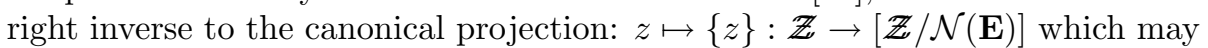
be taken to be of linear growth.

Composing, for any fixed $\xi \in \mathcal{K}_{0}$, there is a continuous selection $\mathbf{C}=\mathbf{C}_{\xi}$ : $\mathcal{V} \rightarrow \mathbb{Z}$ satisfying

$$
\mathbf{L}_{[T]} g+\mathbf{E}[\mathbf{C} g]=\xi
$$

and a linear growth condition: $|\mathbf{C} g|_{\mathcal{Z}}=\mathcal{O}\left(|g|_{\boldsymbol{\nu}}\right)$. It is an important point that $\mathbf{C}$ depends only on the range inclusion (4.29) and not at all on $\mathbf{F}$.

We then consider the map: $\mathbb{Z} \rightarrow \mathbb{Z}$ given by

$$
\mathbf{C G}_{\mathbf{F}}: z \mapsto g:=\mathbf{G}_{\mathbf{F}} z \mapsto \hat{z}:=\mathbf{C} g
$$

and note that this can be restricted to an invariant ball by using the linear growth of $\mathbf{C}$ and the sublinear growth condition (4.28). Using $\left(\mathbf{H}_{1}\right)$ (iii), the Schauder Fixpoint Theorem applies to give the desired result since using a fixpoint $\hat{z}$ of $\mathbf{C}_{\xi} \mathbf{G}$ in (3.15) just gives $\mathbf{T}_{\mathbf{F}} \hat{z}=\xi$.

Our intention here is to use, for the analysis of the approximately reachable set, a modified version of the argument used above for the exactly reachable set. We already have Lemma 2 and have noted that the real problem is to determine

\footnotetext{
${ }^{3}$ In the previous papers $\mathbb{U}_{a d}=\mathbb{U}$ was a Banach space with $\mathbf{B}$ linear. The modification to the present formulation requires no essential change in the ideas at this point since we can topologize $\mathcal{Z}$ by identification with $\mathbb{U} / \mathcal{N}(\mathbf{B})$ when $\mathbf{B}$ is linear.
} 
when $\overline{\mathcal{K}}_{0} \subset \overline{\mathcal{K}}_{\mathbf{F}}$, which is just the question $(\mathbf{Q})$ of the Introduction. It is not difficult to see, much as for (4.27), that we have

$$
\overline{\mathcal{K}}_{\mathbf{F}}=\bigcap_{\varepsilon>0}\{\xi \in \mathcal{X}: \Phi(\xi, \varepsilon) \neq \emptyset\}
$$

where $\Phi(\xi, \varepsilon):=\left\{z \in \mathbb{Z}_{a d}: z \in \mathcal{C}(\mathbf{G} z ; \xi, \varepsilon)\right\}$. Our argument, then, is to show that the set-valued map: $z \mapsto C(\mathbf{G} z ; \xi, \varepsilon)$ has a fixpoint (so $\Phi(\xi, \varepsilon)$ is nonempty) for $\xi \in \mathcal{K}_{0}$ and all $\varepsilon>0$. We take the inclusion $\mathcal{K}_{0} \subset \overline{\mathcal{K}}_{g}$ of (3.20) as a starting point but, again as noted, we will be forced to strengthen this.

The critical difficulty with this program is that (4.29), with $\mathcal{Z}_{a d}$ a Banach space, was essential for the applicability of the theorems used above to obtain the essential continuity and linear growth for $\mathbf{C}_{\xi}$ and so, with the growth condition (4.28), existence of an invariant ball for the mapping $\mathbf{C}_{\xi} \mathbf{G}_{\mathbf{F}}$. The various results in this paper correspond to ways of handling this difficulty.

We have already made our third basic observation: for present purposes we may not only fix $\xi$ (arbitrary $\xi \in \mathcal{K}_{0}$ ) but also $\varepsilon$ (arbitrary $\varepsilon>0$ ): now considering $\mathbf{C}=\mathbf{C}_{\xi, \varepsilon}: \mathcal{G} \rightarrow \mathbb{Z}_{a d}$, we weaken (4.30) to require only that

$$
\mathbf{C}_{\xi, \varepsilon}(g) \in \mathcal{C}(g ; \xi, \varepsilon) \text { so }\left|\xi-\left(\mathbf{L}_{[T]} g+\mathbf{E}[\mathbf{C} g]\right)\right|_{\mathcal{X}} \leq \varepsilon .
$$

One easily sees that having $\mathcal{C}(g ; \xi, \varepsilon)$ nonempty (for each $\xi \in \mathcal{K}_{0}$ and each $\varepsilon>0$ ) is precisely equivalent to the inclusion $\mathcal{K}_{0} \subset \overline{\mathcal{K}}_{g}$. Given this and the compactness of $\mathbf{G}_{\mathbf{F}}$, it is not too difficult to construct $\mathbf{C}=\mathbf{C}_{\xi, \varepsilon}$ continuous giving (4.33) for each $g \in \mathcal{G}_{*}$. Unfortunately, without strengthening this condition we cannot obtain a growth rate for $\mathbf{C}=\mathbf{C}_{\xi, \varepsilon}$ which, with (4.28), would give a bounded invariant set. (Under the strong assumption that $\mathbf{E} \mathcal{G}_{*}$ is known to be precompact in $\mathcal{X}$, we do, however, have Theorem 6, below.) Our basic approach is embodied in the following theorems.

Theorem 2. Assume $\left(\mathbf{H}_{1}\right)$. Let $\mathcal{Z}_{a d}$ be convex and, for some $\xi \in \mathcal{X}$ and $\varepsilon>0$, assume that there is some $R=R(\xi, \varepsilon)$ such that

$$
\beta(R ; \xi, \varepsilon)<R .
$$

Then there is some $\bar{z} \in \mathcal{Z}_{\text {ad }}$ (with $|\bar{z}| \leq R$ ) such that $\left|\xi-\mathbf{T}_{\mathbf{F}} \bar{z}\right| \leq 2 \varepsilon$.

Proof. By $\left(\mathbf{H}_{1}\right)$ we have $\mathbf{G}, \mathbf{L}, \mathbf{T}_{\mathbf{F}}$ continuous so there is no loss of generality in taking $\mathcal{Z}_{\text {ad }}$ closed in $\mathcal{Z}$ (i.e., replacing it by its closure). Now define

$$
\mathcal{Z}_{R}:=\left\{z \in \mathcal{Z}_{a d}:|z| \leq R\right\}, \quad \mathcal{G}_{R}:=\mathbf{G} \mathcal{Z}_{R}=\left\{\mathbf{G} z: z \in \mathbb{Z}_{R}\right\}
$$

Note that (i) $\mathcal{Z}_{R}$ is closed and convex in $\mathcal{Z}$, (ii) $\mathcal{G}_{R}$ is precompact in $\mathcal{V}$ by $\left(\mathbf{H}_{1}\right)$ (iii), and (iii) $\mathcal{C}_{R}(g):=\mathcal{Z}_{R} \cap \mathcal{C}(g ; \xi, \varepsilon)$ is nonempty for each $g \in \mathcal{G}_{R}$ since $\nu(g) \leq \beta(R)<R$. By (ii), we can find a finite set of "centers" $\left\{g_{j}: j=1, \cdots, J\right\}$ such that

$$
\min _{j}\left\{\left|g-g_{j}\right|\right\} \leq \delta:=\varepsilon / 2\left\|\mathbf{L}_{[T]}\right\| \text { for each } g \in \mathcal{G}_{R}
$$


and by (iii), we can find $z_{j} \in \mathcal{C}_{R}\left(g_{j}\right)$ for each $j$. A standard construction gives a continuous partition of unity subordinate to the covering of $\mathcal{G}_{R}$ by $2 \delta$-balls centered at $\left\{g_{j}\right\}$, i.e., continuous scalar functions $\varphi_{j}$ on $\mathcal{V}$ such that

$$
\varphi_{j} \geq 0, \quad \sum \varphi_{j}(g)=1 \text { for } g \in \mathcal{G}_{R}, \quad \varphi_{j}(g)>0 \Longrightarrow\left|g-g_{j}\right| \leq 2 \delta .
$$

We now define $\mathbf{C}=\mathbf{C}_{\xi, \varepsilon}$ by

$$
\mathbf{C} g:=\sum \varphi_{j}(g) z_{j}
$$

and note that this gives $\mathbf{C}: \mathcal{G}_{R} \rightarrow \mathbb{Z}_{R}$ since $\mathbb{Z}_{R}$ is convex. Clearly $\mathbf{C}$ is continuous and, as each $z_{j} \in \mathcal{C}\left(g_{j}\right)$, a simple computation from (3.22) gives

$$
\left|\xi-\left[\mathbf{L}_{[T]} g+\mathbf{E} \mathbf{C} g\right]\right|_{\mathcal{X}} \leq 2 \varepsilon \quad \text { for each } g \in \mathcal{G}_{R}
$$

From $\left(\mathbf{H}_{1}\right)$ we have $\mathbf{C G}: \mathbb{Z}_{R} \rightarrow \mathbb{Z}_{R}$ continuous and compact so, applying the Schauder Fixpoint Theorem, there is a fixpoint $\bar{z} \in \mathbb{Z}_{R}$, i.e., we have $\mathbf{C} \bar{g}=\bar{z}$ for $\bar{g}=\mathbf{G} \bar{z}$. Putting $g=\bar{g}$ gives $\mathbf{L}_{[T]} g+\mathbf{E} \mathbf{C} g=\mathbf{L}_{[T]} \mathbf{G} \bar{z}+\mathbf{E} \bar{z}=: \mathbf{T}_{\mathbf{F}} \bar{z}$ so (4.37) gives $\left|\xi-\mathbf{T}_{\mathbf{F}} \bar{z}\right| \leq 2 \varepsilon$ as desired.

THEOREM 3. Assume $\left(\mathbf{H}_{1}\right)$ with (4.28) and that $\mathbb{Z}_{\text {ad }}$ is convex. Now suppose, for some $\xi \in \mathcal{K}_{0}$ and each $\varepsilon>0$, that one has a growth rate

$$
\nu(g) \leq \tilde{C}_{0}+\tilde{C}_{1}|g|_{\mathcal{w}}^{\tilde{r}} \quad \text { for } g \in \mathcal{G}_{*}
$$

with $\tilde{r}<1 / \bar{r}$. (Here, $\nu$ is defined as in $(3.23)$; the numbers $\tilde{C}_{0}, \tilde{C}_{1}, \tilde{r}$ may depend on $\xi, \varepsilon$.) Then one has $\xi \in \overline{\mathcal{K}}_{\mathbf{F}}$.

Proof. Substituting (4.28) in (4.38) gives

$$
\beta(R) \leq \tilde{C}_{0}+\tilde{C}_{1}\left[C_{0}+C_{1} R^{\bar{r}}\right]^{\tilde{r}}=\mathcal{O}\left(R^{\bar{r} \tilde{r}}\right)=o(R)
$$

as $\bar{r} \tilde{r}<1$. Hence one can always find $R=R(\xi, \varepsilon)$ for which $\beta(R)<R$ so Theorem 2 applies.

Note that if we have this for every $\xi \in \mathcal{K}_{0}$ and also have the hypotheses of Lemma 2, then we have shown, as in the title, invariance of the approximately reachable set under the nonlinear perturbation $\mathbf{F}$, i.e., $\overline{\mathcal{K}}_{\mathbf{F}}=\overline{\mathcal{K}}_{0}$.

The condition (4.28) is the same growth condition for the nonlinearity as was imposed in Theorem 1. Note also that the condition (4.38) implies, in particular, that $\nu$ is finite and so quantitatively strengthens the simple requirement of approximate reachability for each (1.4):

$$
\xi \subset \overline{\mathcal{K}}_{g}=\mathbf{L}_{[T]} g+\overline{\mathcal{K}}_{0} \text { for each } g \in \mathcal{G}_{*}:=\left\{\mathbf{G}_{\mathbf{F}} z: z \in \mathcal{Z}_{a d}\right\}
$$

which, for every $\xi \in \mathcal{K}_{0}$, would just be (3.21). We can also use this approach - more-or-less unchanged, with proper formulation - in a somewhat more complicated nonlinear setting in which the linear operator $\mathbf{A}$ is also taken to depend on $x, u$. Without developing this further at the present time, we provide 
the formulation here in the hope that it will also add to the reader's insight into the essential aspects of the original argument above. Clearly one might continue to extend the same basic ideas.

We now suppose that we wish to analyze approximate reachability for an equation of the form

$$
\dot{x}=\mathbf{A}(x, u) x+f(x, u)+\mathbf{B} u, \quad u \in \mathbb{U}_{a d}
$$

by comparison with similar considerations for the family of (presumably simpler) equations (1.4); now, $\mathbf{A}$ ranges over a suitable set $\mathcal{A l}$ of linear operators and $g$ ranges over $\mathcal{G}$. As above, we assume that $\mathbf{A}\left(x_{u}, u\right) \in \mathscr{A l}$ and $f\left(x_{u}, u\right) \in \mathcal{G}$ for each solution $x_{u}$ of (4.40) as $u$ ranges over $\mathbb{U}_{a d}$ and that that solutions are here also taken in $\mathbb{X}:=C([0, T] \rightarrow \mathcal{X})$ where $\mathcal{X}$ is the state space. We assume that there are well-defined maps

$$
\begin{array}{rcl}
\mathbf{M}: & \boldsymbol{U}_{a d} \rightarrow \mathcal{A} & : u \mapsto \mathbf{A}\left(x_{u}, u\right), \\
\mathbf{L}_{[T]}: & \boldsymbol{U}_{a d} \times \mathcal{A} \times \mathcal{G} \rightarrow \mathcal{X} & :[u, \mathbf{A}, g] \mapsto x_{u}(T)
\end{array}
$$

given by (4.40) and (1.4), respectively. We will take $\mathbb{U}_{a d}$ to be a convex subset of a suitable Banach space and take $\mathcal{A}, \mathcal{G}$ to be compact Hausdorff spaces; we assume the topologies can be taken so that $\mathbf{L}_{[T]}$ and $\mathbf{M}$ are continuous. Much as before, we then introduce

$$
\begin{aligned}
\mathcal{C}(\mathbf{A}, g ; \xi, \varepsilon) & =\left\{u \in \mathcal{U}_{a d}:\left|\xi-\mathbf{L}_{[T]}[u, \mathbf{A}, g]\right|<\varepsilon\right\} \\
\nu(\mathbf{A}, g ; \xi, \varepsilon) & =\inf \{|u|: u \in \mathcal{C}(\mathbf{A}, g ; \xi, \varepsilon)\} \\
R(\xi, \varepsilon) & =\sup \{\nu(\mathbf{A}, g ; \xi, \varepsilon): \mathbf{A} \in \mathcal{A}, g \in \mathcal{G}\} .
\end{aligned}
$$

For $\nu(\mathbf{A}, g ; \xi, \varepsilon)$ to be finite just means that $\mathcal{C}(\mathbf{A}, g ; \xi, \varepsilon)$ is nonempty and this will be the case, for the given $\xi \in \mathcal{X}$ and every $\varepsilon>0$, if and only if this $\xi$ is approximately reachable for (1.4) using this $\mathbf{A} \in \mathcal{A}$. This, of course, would not be sufficient in itself to ensure finiteness of $R(\xi, \varepsilon)$ unless, for example, one were to know that $\nu(\cdot ; \xi, \varepsilon)$ would be upper semicontinuous on the (assumed) compact set $\mathcal{A} \times \mathcal{G}$.

THEOREM 4. Let the setting be as assumed above and suppose, for some fixed $\xi \in \mathcal{X}$, that we have $R(\xi, \varepsilon)<\infty$ for each $\varepsilon>0$. Then this $\xi$ is approximately reachable for $(4.40)$.

Proof. Choose some $R>R(\xi, \varepsilon)$ and restrict attention to the ball $\boldsymbol{U}_{R}:=$ $\left\{u \in \mathcal{U}_{a d}:|u| \leq R\right\}$. We can then construct a continuous map $\mathbf{C}=\mathbf{C}_{\xi, \varepsilon}$ : $[\mathcal{A l} \times \mathcal{G}] \rightarrow \mathbb{U}_{R}$ such that, corresponding to (4.33), we have

$$
\left|\xi-\mathbf{L}_{[T]}[\mathbf{C}(\mathbf{A}, g), \mathbf{A}, g]\right|_{\mathcal{X}} \leq 2 \varepsilon
$$

for each $[\mathbf{A}, g] \in \mathscr{A} \times \mathcal{G}$. We omit detail since this is much as in the proof of Theorem 2 - what corresponds to (4.36) is that we can find, for each $[\mathbf{A}, g]$, a control $\tilde{u} \in \mathbb{U}_{R} \cap \mathcal{C}(\mathbf{A}, g ; \xi, \varepsilon)$ and then a neighborhood $\mathcal{N}=\mathcal{N}(\mathbf{A}, g)$ on 
which the continuous function $\mathbf{L}_{[T]}[\tilde{u}, \cdot]$ varies by no more than $\varepsilon$ in $\mathcal{X}$-norm; there is then a finite subcovering by such neighborhoods, and one can find a corresponding partition of unity.

Note that the continuity of $\mathbf{G}$ and the compactness of $\mathcal{A} \times \mathcal{G}$ make the composition $\mathbf{C G}$ a continuous, compact selfmap of the bounded convex set $\boldsymbol{U}_{R}$. The Schauder Fixpoint Theorem applies to ensure a fixpoint $\hat{u}$ for which (4.40) gives $\left|\xi-x_{\hat{u}}(T)\right| \leq 2 \varepsilon$. This, for each $\varepsilon>0$, ensures the approximate reachability of this $\xi$ for (4.40).

\section{$5 \quad$ Further results}

Having verified $\left(\mathbf{H}_{1}\right)$, the principal problem in applying the general results above is, of course, the difficulty in verifying a condition such as (4.38) to enable one to restrict attention to some $\mathscr{Z}_{R}$. There are, however, certain cases in which one can proceed. We note one in which $\mathbb{Z}_{a d}$ is the whole Banach space $\mathcal{Z}$ and another in which the nonlinearity $\mathbf{F}$ gives only a compactly restricted perturbation of the final value.

Since we only consider $\nu\left(g ; \xi_{0}, \varepsilon\right)$ for $\xi_{0} \in \mathcal{K}_{0}$ so $\xi_{0}=\mathbf{T} z_{0}$, we can introduce

$$
\bar{\nu}(\bar{\xi} ; \varepsilon):=\inf \left\{\left|z^{\prime}\right|: z^{\prime} \in\left[z_{0}-\mathbb{Z}_{a d}\right],\left|\bar{\xi}-\mathbf{T} z^{\prime}\right| \leq \varepsilon\right\}
$$

and have $\nu\left(g ; \mathbf{T} z_{0}, \varepsilon\right)=\bar{\nu}\left(\mathbf{L}_{[T]} g, \varepsilon\right)$. Observe that if we consider $\mathbb{Z}_{a d}=\mathscr{Z}$, then scaling gives $\bar{\nu}(\lambda \bar{\xi}, \varepsilon)=\lambda \bar{\nu}(\bar{\xi}, \varepsilon / \lambda)$ so (4.38) is equivalent to requiring that

$$
\nu_{*}^{\vartheta}(\bar{\xi}):=\limsup _{\varepsilon \rightarrow 0}\left\{\inf _{z}\left\{\varepsilon^{-(1-\vartheta)}|z|: z \in \mathbb{Z} \text { and }|\bar{\xi}-\mathbf{E} z|_{\mathcal{X}} \leq \varepsilon\right\}\right\}
$$

should be bounded for $\bar{\xi} \in\left\{\mathbf{L}_{[T]} g: g\right.$ in some $\mathcal{V}$-bounded subset of $\left.\mathcal{G}_{*}\right\}$. It is possible to show that $\nu_{*}^{\vartheta}$ is actually a norm intermediate between the $\mathcal{X}$-norm on $\overline{\mathcal{K}}_{0}(\vartheta=0)$ and the obvious induced norm: $|\xi|_{1}:=\inf \{|z|: \mathbf{T} z=\xi\}$ on $\mathcal{K}_{0}$ $(\vartheta=1)$. Thus, finiteness of $\nu_{*}^{\vartheta}\left(\mathbf{L}_{[T]} g\right)$ is a stronger condition than just requiring $\mathbf{L}_{[T]} g \in \overline{\mathcal{K}}_{0}$ but is weaker than the exact reachability condition of Theorem 2 , i.e., that $\mathbf{L}_{[T]} g \in \mathcal{K}_{0}$. We will not analyze $\nu_{*}^{\vartheta}$ directly but will, instead, use the established theory Banach space interpolation (cf., e.g., [4]).

TheOREm 5. Suppose $\mathcal{Z}_{a d}=\mathcal{Z}_{\text {so }} \overline{\mathcal{K}}_{0}=: \mathcal{X}_{0}$ is a (closed) subspace of $\mathcal{X}$. Assume $\left(\mathbf{H}_{1}\right)$ and suppose ${ }^{4}$ that, for some $\vartheta>0$, one has

$$
\mathbf{L}_{[T]} g \in \mathcal{X}_{\vartheta} \quad \text { for each } g \in \mathcal{V}
$$

where $\mathcal{X}_{\vartheta}$ is an interpolation space $\left[\mathcal{X}_{0}, \mathcal{X}_{1}\right]_{\vartheta}$ with $\mathcal{X}_{1}:=\mathcal{K}_{0}$ (taken with the norm: $|\xi|_{1}:=\inf \left\{|z|: \mathbf{E} z=\xi\right.$ for $\left.\xi \in \mathcal{X}_{1}=\mathcal{K}_{0}\right\}$; note that here $|z|$ is the

\footnotetext{
${ }^{4}$ Given $\mathbf{F}$, the hypothesis (5.41) with $\vartheta>\bar{r}$ is somewhere between taking $\vartheta=0$, which just reduces to the (inadequate) hypothesis (4.39), and taking $\vartheta=1$ which is equivalent to the exact reachability inclusion (4.29) used in Theorem 1. Note that it is easiest to obtain (5.41) if one takes $\boldsymbol{V}$ as small as possible consistent with $\left(\mathbf{H}_{1}\right)$.
} 
$\mathcal{Z}$-norm $)$ and $\mathcal{X}_{0}:=\overline{\mathcal{K}}_{0}$ with the $\mathcal{X}$-norm. Let $\mathbf{F}$ be a nonlinearity satisfying the growth condition (4.28) for some $\bar{r}<\vartheta$. Then the approximately reachable set $\overline{\mathcal{K}}_{\mathbf{F}}$ is precisely $\overline{\mathcal{K}}_{0}$.

Proof. Since (5.41) gives $\mathbf{L}_{[T]} \boldsymbol{V} \subset \mathcal{X}_{1} \subset \mathcal{X}_{0}$ and these are subspaces, we have (4.26) so $\overline{\mathcal{K}}_{\mathbf{F}} \subset \overline{\mathcal{K}}_{0}$ by Lemma 2 . As usual, we are primarily concerned with the complementary inclusion.

While there are various possible interpolation functors, the extremal property of the $K$-functor (see, e.g., Theorem 3.9.1 of [4]) gives a uniform estimate:

$$
s^{-\vartheta} K(s ; \xi) \leq C|\xi|_{\vartheta} \quad\left(s>0, \xi \in \mathcal{X}_{\vartheta}\right)
$$

( $C$ depending on the choice of $|\cdot|_{\vartheta}$ ) where the function $K(\cdot)$ is defined by

$$
\begin{aligned}
K(s ; \xi) & :=\inf \left\{\left|\xi_{0}\right|_{\mathcal{X}}+s\left|\xi_{1}\right|_{1}: \xi_{0}+\xi_{1}=\xi, \xi_{1} \in \mathcal{X}_{1}\right\} \\
& =\inf \{s|z|+|\xi-\mathbf{E} z|: z \in \mathcal{Z}\} .
\end{aligned}
$$

Fixing $\varepsilon>0$, define

$$
\omega(\nu)=\omega(\nu ; \varepsilon):=\left[C \varepsilon^{-(1-\vartheta)}\right]^{1 / \vartheta} \nu^{1 / \vartheta} .
$$

For any $\xi \in \mathcal{X}_{\vartheta}$ set $\nu:=|\xi|_{\vartheta}$ and consider $s=\varepsilon / \omega$ in (5.42), (5.43) with $\omega>\omega(\nu)$. that

From (5.42) this gives $K(s ; \xi)<\varepsilon$ so, from (5.43), there exists $z \in \mathcal{Z}$ such

$$
|\xi-\mathbf{E} z|<\varepsilon, \quad|z|<\omega .
$$

(Here the first is the $\mathcal{X}$-norm while the second is the $\mathcal{Z}$-norm.) Since we may take $\omega$ arbitrarily close to $\omega(\nu)$, this shows:

$$
\inf \{|z|:|\xi-\mathbf{E} z| \leq \varepsilon\} \leq\left[C \varepsilon^{-(1-\vartheta)}\right]^{1 / \vartheta}|\xi|_{\vartheta}^{1 / \vartheta}
$$

for $\xi \in \mathcal{X}_{\vartheta}$.

Note that (5.41) implies, by the Closed Graph Theorem, continuity of $\mathbf{L}_{[T]}$ as a linear operator from $\mathcal{V}$ to $\mathcal{X}_{\vartheta}$, i.e., existence of a constant $\bar{C}$ such that $\left|\mathbf{L}_{[T]} g\right|_{\vartheta} \leq \bar{C}|g|\left(|g|\right.$ is the $\boldsymbol{V}$-norm). Now fix $\xi_{0}=\mathbf{E} z_{0} \in \mathcal{K}_{0}=\mathcal{X}_{1}$ and, letting $\xi=\mathbf{L}_{[T]} g$ in (5.44), note that $|\xi-\mathbf{E} z| \leq \varepsilon$ if and only if

$$
\left|\xi_{0}-\left[\mathbf{L}_{[T]} g+\mathbf{E}\left(z_{0}-z\right)\right]\right|_{\mathcal{X}} \leq \varepsilon \text { so } z_{0}-z=: z^{\prime} \in \mathcal{C}\left(g ; \xi_{0}, \varepsilon\right) .
$$

From (5.44), any $\omega>\omega\left(\left|\mathbf{L}_{[T]} g\right|_{\vartheta}\right)$ can be used to estimate $z^{\prime}$ so

$$
\begin{aligned}
\nu\left(g ; \xi_{0}, \varepsilon\right) & =\inf \left\{\left|z^{\prime}\right|: z^{\prime}=z_{0}-z \in \mathcal{C}\left(g ; \xi_{0}, \varepsilon\right)\right\} \\
& \leq\left|z_{0}\right|+\inf \left\{|z|: z_{0}-z \in \mathcal{C}\left(g ; \xi_{0}, \varepsilon\right)\right\} \\
& \leq\left|z_{0}\right|+\left[C \varepsilon^{-(1-\vartheta)}\right]^{1 / \vartheta}[\bar{C}|g|]^{1 / \vartheta}
\end{aligned}
$$

We recognize this as (4.38) with $\tilde{r}=1 / \vartheta$; the assumption $\vartheta>\bar{r}$ gives $\tilde{r}<1 / \bar{r}$. Thus Theorem 3 applies to show $\mathcal{K}_{0} \subset \overline{\mathcal{K}}_{\mathbf{F}}$ and one has the desired invariance: $\overline{\mathcal{K}}_{\mathbf{F}}=\overline{\mathcal{K}}_{0}$. 
Corollary. Suppose $\mathcal{V}_{0}$ is any space for which $\mathbf{L}_{[T]}: \mathcal{V}_{0} \rightarrow \mathcal{X}$ is continuous and $\boldsymbol{V}_{1}$ is any space for which the exact reachability condition holds: $\left\{\mathbf{L}_{[T]} g\right.$ : $\left.g \in \mathcal{V}_{1}\right\} \subset \mathcal{K}_{0}$. Assume $\left(\mathbf{H}_{1}\right)$ with $\boldsymbol{V}$ taken as $\mathcal{V}_{\vartheta}:=\left[\mathcal{V}_{0}, \mathcal{V}_{1}\right]_{\vartheta}$ for some $\vartheta>\bar{r}$; assume (4.26) and (4.28). Then one has $\overline{\mathcal{K}}_{\mathbf{F}}=\overline{\mathcal{K}}_{0}$.

Proof. Let $\mathbf{L}_{[T]}^{0}$ be $\mathbf{L}_{[T]}: \mathcal{V}_{0} \rightarrow \mathcal{X}_{0}:=\overline{\mathcal{K}}_{0}$ and let $\mathbf{L}_{[T]}^{1}$ be $\mathbf{L}_{[T]}: \mathcal{V}_{1} \rightarrow \mathcal{X}_{1}:=$ $\mathcal{K}_{0}$; the latter is bounded by the Closed Graph Theorem since $\mathbf{L}_{[T]} \mathcal{\nu}_{1} \subset \mathcal{X}_{1}$. Then boundedness of $\mathbf{T}^{\vartheta}: \mathcal{V}_{\vartheta} \rightarrow \mathcal{X}_{\vartheta}:=\left[\mathcal{X}_{0}, \mathcal{X}_{1}\right]_{\vartheta}$ follows from interpolation theory [4] and Theorem 5 applies.

A somewhat modified fixpoint argument provides our final result.

Theorem 6. Assume $\left(\mathbf{H}_{1}\right)$ (i),(ii) and (4.26). Let $\mathcal{Z}_{\text {ad }}$ be convex and closed in $\mathcal{Z}$ and assume we have (3.21) for each $g \in \mathcal{G}_{*}:=\left\{\mathbf{G}_{\mathbf{F}} z: z \in \mathbb{Z}_{a d}\right\}$. Finally, we assume that $\mathbf{L}_{[T]} \mathcal{G}_{*}:=\left\{\mathbf{L}_{[T]} \mathbf{G}_{\mathbf{F}} z: z \in \mathcal{Z}_{a d}\right\}$ is precompact in $\mathcal{X}$. Then, one has $\overline{\mathcal{K}}_{\mathbf{F}}=\overline{\mathcal{K}}_{0}$.

Proof. By Lemma 2 we have $\overline{\mathcal{K}}_{\mathbf{F}} \subset \overline{\mathcal{K}}_{0}$ and, as earlier, need only show $\xi \in \overline{\mathcal{K}}_{\mathbf{F}}$ for each fixed $\xi=\xi_{0} \in \mathcal{K}_{0}$.

Note that (3.21) just means that $\left[\xi_{0}-\mathbf{L}_{[T]} g\right] \in \overline{\mathcal{K}}_{0}$ and this, for each $g \in \mathbf{G}_{*}$, means $\left[\xi_{0}-\mathbf{L}_{[T]} \mathbf{G}_{*}\right] \subset \overline{\mathcal{K}}_{0}$. As $\overline{\mathcal{K}}_{0}:=\overline{\mathbf{E}}_{\mathcal{Z}_{a d}}$ is convex, since $\mathscr{Z}_{a d}$ is and $\mathbf{E}$ is linear, we have $\mathcal{X}_{*}:=\overline{c o}\left(\xi_{0}-\mathbf{L}_{[T]} \mathcal{G}_{*}\right) \subset \overline{\mathcal{K}}_{0}$. On the other hand, we note that $\mathcal{X}_{0}:=\overline{c o}\left(\mathbf{L}_{[T]} \mathcal{G}_{*}\right)$ is convex and is compact by the precompactness assumption so $\mathcal{X}_{*}$ is compact since we easily see $\mathcal{X}_{*}:=\xi_{0}-\mathcal{X}_{0}$.

Given any $\varepsilon>0$, one can then find a finite covering of $\mathcal{X}_{*}$ by $\varepsilon$-balls which may be taken centered at $\left\{\xi_{j}: j=1, \cdots, J\right\}$ with each $\xi_{j} \in \mathcal{K}_{0} \cap \mathcal{X}_{*}$ so there exist $z_{j} \in \mathbb{Z}_{a d}$ such that $\mathbf{E} z_{j}=\xi_{j}$. As in the proof of Theorem 2, we can find a continuous partition of unity subordinate to this covering:

$$
\varphi_{j} \geq 0, \quad \sum \varphi_{j} \equiv 1 \text { on } \mathcal{X}_{*}, \quad \varphi_{j}(\xi) \neq 0 \Longrightarrow\left|\xi-\xi_{j}\right|<\varepsilon .
$$

and then define $\mathbf{C}=\mathbf{C}_{\varepsilon}$ by

$$
\mathbf{C} \xi:=\sum \varphi_{j}(\xi) z_{j}
$$

noting that $\mathbf{C} \xi \in \mathbb{Z}_{a d}$ for $\xi \in \mathcal{X}_{*}$ by the assumed convexity of $\mathbb{Z}_{a d}$. Clearly $\mathbf{C}: \mathcal{X}_{*} \rightarrow \mathbb{Z}_{a d}$ is continuous and, as earlier, a simple computation shows that

$$
|\xi-\mathbf{E C} \xi| \leq \varepsilon \quad \text { for } \xi \in \mathcal{X}_{*}
$$

As already noted, for any $z \in \mathbb{Z}_{a d}$ we have $\left[\xi-\mathbf{L}_{[T]} \mathbf{G} z\right] \in \mathcal{X}_{*}$ so the map:

$$
\xi \mapsto z:=\mathbf{C} \xi \mapsto\left[\xi-\mathbf{L}_{[T]} \mathbf{G} z\right]
$$

is a continuous selfmap of the compact, convex set $\mathcal{X}_{*}$.

By the Schauder Fixpoint Theorem this map has a fixpoint $\bar{\xi}$ so, setting $\bar{z}:=\mathbf{C} \bar{\xi} \in \mathcal{Z}_{\text {ad }}$ we have $\bar{\xi}=\xi-\mathbf{L}_{[T]} \mathbf{G} \bar{z}$. Using (5.46), we have $\left|\xi-\mathbf{T}_{\mathbf{F}} \bar{z}\right|_{\mathcal{X}}=$ $\left|\xi-\left[\mathbf{L}_{[T]} \mathbf{G} \bar{z}+\mathbf{T} \bar{z}\right]\right|_{\mathcal{X}}=|\bar{\xi}-\mathbf{E} \bar{z}|_{\mathcal{X}} \leq \varepsilon$. Since this is possible for each $\varepsilon>0$, we have $\xi \in \overline{\mathcal{K}}_{\mathbf{F}} \subset \overline{\mathcal{K}}_{\mathbf{F}}$. Since that holds for each $\xi \in \mathcal{K}_{0}$, we have $\overline{\mathcal{K}}_{0} \subset \overline{\mathcal{K}}_{\mathbf{F}}$. 


\section{Approaches to the hypotheses}

Finally, we consider the verification of the abstract hypothesis $\left(\mathbf{H}_{1}\right)$ for more concrete settings - e.g., such as those presented in $\S 2$. We begin by noting that in connection with $\left(\mathbf{H}_{1}\right)$ (ii), (iii) there may be an advantage in introducing an auxiliary function space $\mathcal{Y}$.

Lemma 3. Suppose $\mathcal{Z}$ embeds continuously in a space $\mathcal{Y}$ which is compatible with $\boldsymbol{X}$ in the sense that the set $\mathcal{Y} \cap \boldsymbol{X}$ is dense both in $\mathcal{Y}$ and in $\boldsymbol{X}$ with

$$
y_{k} \in \mathcal{Y} \cap \boldsymbol{x}, \quad y_{k} \stackrel{\boldsymbol{y}}{\rightarrow} \hat{y}, \quad y_{k} \stackrel{\boldsymbol{x}}{\rightarrow} \hat{x} \Longrightarrow \hat{y}=\hat{x} \in \boldsymbol{Y} \cap \boldsymbol{X} .
$$

Assume that, in addition to $\left(\mathbf{H}_{1}\right)(\mathrm{i})$, the linear operator $\mathbf{L}$ is continuous from $\mathcal{V}$ to $\mathcal{Y}$ and that the nonlinear map $\mathbf{F}$ is Lipschitzian from $\mathcal{Y}$ to $\mathcal{V}$ with Lipschitz constant $C$ such that $C\|\mathbf{L}\| \boldsymbol{v} \rightarrow \boldsymbol{\nu}=: \vartheta<1$. Then:

(i) One has $\left(\mathbf{H}_{1}\right)$ (ii) with $\mathbf{G}_{\mathbf{F}}$ Lipschitzian.

(ii) If (in addition to the original hypotheses) one has a growth condition

$$
|\mathbf{F} y|_{\boldsymbol{\nu}} \leq a_{0}+a_{1}|y|_{\grave{y}}^{\bar{y}}
$$

for $\mathbf{F}$, then $\mathbf{G}_{\mathbf{F}}$ satisfies the growth condition (4.28).

(iii) If (in addition to the original hypotheses) one has

$$
\begin{aligned}
& \left\{\mathbf{F}(\mathbf{L} g+z): g \in \mathcal{G}_{0}, z \in \mathcal{Z}_{0}\right\} \text { precompact in } \mathcal{V} \\
& \text { for each compact subset } \mathcal{G}_{0} \subset \mathcal{V},
\end{aligned}
$$

then $\mathbf{G}_{\mathbf{F}}\left(\mathbb{Z}_{0}\right)$ is precompact in $\mathcal{V}$. (Note that if (6.49) holds for each bounded subset $\mathcal{Z}_{0}$ of $\mathcal{Z}$, then (iii) gives $\left(\mathbf{H}_{1}\right)$ (iii); a sufficient condition for this, in view of (i), is that the embedding $\mathbb{Z} \hookrightarrow \mathcal{Y}$ is compact.)

Proof. Under the assumption $\vartheta<1$, the map: $y \mapsto[\mathbf{L F} y+z]$ is a contraction on $\mathbb{D}$ so there is a unique fixpoint $y=Y(z)$, the solution of (3.15). One easily obtains the Lipschitz constant $1 /(1-\vartheta)$ for the solution map $Y(\cdot): z \mapsto y$ : $\mathcal{Y} \rightarrow \mathcal{Y}$ and composing this with the Lipschitzian maps $\mathbb{Z} \hookrightarrow \mathcal{Y}$ on one side and $\mathbf{F}$ on the other gives the desired Lipschitz continuity for $\mathbf{G}_{\mathbf{F}}: z \mapsto \mathbf{F} y: \mathcal{Z} \rightarrow \boldsymbol{V}$. This gives linear growth for $Y(\cdot): \mathbb{Z} \rightarrow \mathcal{Y}$ and composing that with (6.48) gives (4.28). The compactness assertion (iii) is an immediate consequence of Lemma 2 in [14], applied to the uniformly contractive family of maps: $g \mapsto \mathbf{F}(\mathbf{L} g+z)$ $\left(z \in \mathbb{Z}_{0}\right)$ whose fixpoints give $\mathbf{G}_{\mathbf{F}}\left(\mathbb{Z}_{0}\right)$.

Note that our treatment of these problems presupposes well-posedness for the equations and, for our application of fixed-point arguments, convexity of $\mathbb{Z}_{\text {ad }}$ and suitable compactness.

We now turn to a somewhat more general consideration of abstract settings giving $\left(\mathbf{H}_{1}\right)$. For this consideration our present concerns are essentially identical 
with those of [15] and we recall the relevant discussion there in providing classes of settings for which the relevant well-posedness and compactness can be verified. For this we suppose $\mathbf{A}(\cdot)$ generates a fundamental solution (evolution system) $\mathbf{S}-$ i.e.,

$$
\begin{array}{ll}
\text { (i) } & \mathbf{S}(t, s) \text { is a bounded linear operator on } \mathcal{X} \text { with } \\
& \|\mathbf{S}(t, s)\| \leq M \text { for } 0 \leq s \leq t \leq T ; \\
\text { (ii) } \quad & \mathbf{S}(t, s) \mathbf{S}(s, r)=\mathbf{S}(t, r) \text { for } 0 \leq r \leq s \leq t \leq T ; \\
\text { (iii) } & \mathbf{S}(t, s) \xi \longrightarrow \xi \text { as } t \rightarrow s+\text { for } \xi \in \mathcal{X} \\
\text { (iv) } & \partial \mathbf{S}(t, s) \xi / \partial t=\mathbf{A}(s) \xi \text { for } t=s \text { and } \xi \in \mathcal{D}=\mathcal{D}(\mathbf{A}(s)) .
\end{array}
$$

This permits us to introduce the notion of a mild solution [9] of (1.2) or (1.4): the linear map $\mathbf{L}$ will be given, in terms of $\mathbf{S}(\cdot)$, by

$$
[\mathbf{L} v](t)=\mathbf{L}_{[t]} v:=\int_{0}^{t} \mathbf{S}(t, s) v(s) d s \text { for } 0 \leq t \leq T
$$

for suitable $v(\cdot)$. In this formulation, (1.1) corresponds to the nonlinear integral equation $^{5}$ (abstract Volterra equation of second kind):

$$
x(t)=\bar{z}(t)+\int_{0}^{t} \mathbf{S}(t, s)[f(s, x(s))+[\mathbf{B}(u)](s)] d s
$$

This is, of course, just the operator equation (3.15).

Now introduce (reflexive) Banach spaces $\mathcal{V}, \mathcal{W}$ and spaces $\mathcal{V}$ and $\mathbb{W}$ of $\mathcal{V}$ - and $\mathcal{W}$-valued functions, respectively, on $[0, T]$. We assume $\mathcal{V}$, WW are compatible with $\mathcal{X}$ in the sense that the set $\mathcal{V} \cap \mathcal{X}$ is dense both in $\mathcal{V}$ and in $\mathcal{X}$ with

$$
v_{k} \in \mathcal{V} \cap \mathcal{X}, \quad v_{k} \stackrel{\mathcal{V}}{\rightarrow} \hat{v}, \quad v_{k} \stackrel{\mathcal{X}}{\rightarrow} \hat{x} \Longrightarrow \hat{v}=\hat{x} \in \mathcal{V} \cap \mathcal{X}
$$

and similarly for $[\mathcal{W}, \mathcal{X}]$. It will thus be possible to make suitable extensions or restrictions of $\mathbf{S}(t, s)$ so, e.g., the formal definition (3.14) may make sense for $v$ in $\mathcal{V}$ or in $\mathbb{W}$.

We note that [15] provides four alternate sets of more concrete conditions on $\mathcal{X}, \mathcal{W}, \mathcal{V}, \mathbf{S}(\cdot \cdot), f(\cdot \cdot)$ under which one can verify $\left(\mathbf{H}_{1}\right)$. For convenience of reference we present these here, converted to our present notation. For this, we take $\mathcal{V}$. WN to have the form

$$
\mathcal{V}:=L^{p}([0, T] \rightarrow \mathcal{V}), \quad \text { WN }:=L^{p^{\prime}}([0, T] \rightarrow \mathcal{W})
$$

and introduce another possible space $\mathcal{Y}$ compatible with $\mathcal{X}$. We assume:

\footnotetext{
${ }^{5}$ Here $\bar{z}(t)$ is the solution of the equation $\dot{z}=\mathbf{A} z$ with the original initial conditions. With our assumption that the problem has been formulated so as to have homogeneous initial conditions, this term vanishes: $\bar{z}=0$.
} 
$\left(\mathbf{C}_{1}\right)$ Let each of the following hold:

(i) $\|\mathbf{S}(t, s)\|_{\mathcal{V} \rightarrow \mathcal{X}} \leq \rho_{\mathcal{v}}(t-s), \quad\|\mathbf{S}(t, s)\|_{\mathcal{W} \rightarrow \mathcal{X}} \leq \rho_{\mathcal{W}}(t-s)$, $\|\mathbf{S}(t, s)\|_{\mathcal{V} \rightarrow \mathcal{Y}} \leq \hat{\rho}_{\mathcal{V}}(t-s), \quad\|\mathbf{S}(t, s)\|_{\mathcal{W} \rightarrow \mathcal{Y}} \leq \hat{\rho}_{\mathcal{W}}(t-s) ;$

(ii) $\left\|\mathbf{S}(t, s)-\mathbf{S}\left(t^{\prime}, s\right)\right\|_{\mathcal{Y} \rightarrow \mathcal{X}},\left\|\mathbf{S}(t, s)-\mathbf{S}\left(t^{\prime}, s\right)\right\|_{\mathcal{W} \rightarrow \mathcal{X}} \leq \varepsilon$ for $0 \leq s \leq t^{\prime}-\varepsilon, t^{\prime}<t \leq T$ with $\varepsilon=\varepsilon(h) \rightarrow 0$ as $h:=t-t^{\prime} \rightarrow 0 ;$

(iii) $|f(t, \eta)|_{\mathcal{V}} \leq \alpha(t)+\beta|\eta|_{\nu}^{\bar{r}} \quad(\bar{r}:=\bar{p} / p<1)$ $\left|\mathbf{S}(t, s)\left[f(s, \eta)-f\left(s, \eta^{\prime}\right)\right]\right|_{\mathcal{Y}} \leq \rho_{\mathcal{Y}}(t-s)\left|\eta-\eta^{\prime}\right|_{\mathcal{Y}}$

where

$$
\rho_{\mathcal{\nu}} \in L^{q}, \quad \rho_{\mathcal{W}} \in L^{q^{\prime}} \quad \hat{\rho}_{\mathcal{\nu}} \in L^{\bar{q}}, \quad \hat{\rho}_{\mathcal{W}} \in L^{\overline{q^{\prime}}}, \quad \alpha \in L^{p}, \quad \rho_{\mathcal{y}} \in L^{1},
$$

with $1<p, p^{\prime}<\infty, 1 \leq \bar{p}<p$, and

$$
1 / p+1 / q=1 / p^{\prime}+1 / q^{\prime}=1 ; 1 / p+1 / \bar{q}, 1 / p^{\prime}+1 / \bar{q}^{\prime} \leq 1+1 / \bar{p} .
$$

To the set of conditions $\left(\mathbf{C}_{1}\right)$ we may adjoin any of four alternative conditions:

$\left(\mathbf{C}_{2}\right)$ Let any one of the following hold:

(i) For some Banach space $\hat{\mathcal{Y}}=\hat{\mathcal{Y}}_{i}$ such that the embedding: $\mathcal{Y} \hookrightarrow \hat{\mathcal{Y}}$ is compact, assume that for small $\delta>0$ there exists $M_{\delta}$ such that

$$
\|\mathbf{S}(t, t-\delta)\|_{\hat{\mathcal{\nu}} \rightarrow \mathcal{Y}} \leq M_{\delta} \quad \text { for } \delta \leq t \leq T .
$$

(ii) For some Banach space $\hat{\mathcal{Y}}=\hat{\mathcal{Y}}_{i i}$ such that the embedding $\hat{\mathcal{Y}} \hookrightarrow \mathcal{Y}$ is compact, strengthen $\left(\mathbf{C}_{1}\right)(\mathrm{i})$ by requiring:

$$
\|\mathbf{S}(t, s)\|_{\mathcal{W} \rightarrow \hat{\mathcal{Y}}} \leq \hat{\rho}_{\mathcal{W}}(t-s) \quad(\hat{\mathcal{Y}} \text { replacing } \mathcal{Y}) .
$$

(iii) For some Banach space $\hat{\mathcal{Y}}=\hat{\mathcal{Y}}_{i i i}$ such that the embedding: $\mathcal{Y} \hookrightarrow \hat{\mathcal{Y}}$ is compact, strengthen the growth condition in $\left(\mathbf{C}_{1}\right)$ (iii) by requiring

$$
|f(t, \zeta)|_{\mathcal{V}} \leq \alpha(t)+\beta|\zeta|_{\hat{\mathcal{Y}}}^{\bar{r}} \text {. }
$$

with $\bar{r}:=\bar{p} / p<1(\hat{\mathcal{Y}}$ replacing $\mathcal{Y})$.

(iv) Take $\mathcal{Y}=\mathcal{X}$ reflexive in $\left(\mathbf{C}_{1}\right)$ and, for some Banach space $\hat{\mathcal{Y}}=\hat{\mathcal{Y}}_{i v}$ such that $\mathcal{X}=\mathcal{Y} \hookrightarrow \hat{\mathcal{Y}}$ is a compact embedding, assume that for each $\mu>0$ there exists $\alpha_{\mu} \in L^{p}$ for which

$$
|\zeta|_{\hat{\mathcal{\nu}}} \leq \mu \Longrightarrow|f(t, \zeta)|_{\nu} \leq \alpha_{\mu}(t) .
$$

Lemma 4. Let $\mathcal{X}, \mathcal{W}, \mathcal{V}$, etc., be as above and assume $\left(\mathbf{C}_{1}\right)$; we will norm $\mathcal{Z}:=\mathbf{L W}$ so $\mathbf{L}$ is an isometry. Then one has $\left(\mathbf{H}_{1}\right)(\mathrm{i})$ and $\left(\mathbf{H}_{1}\right)(\mathrm{ii})$ as well as the growth condition (4.28). If, in addition, we assume $\left(\mathbf{C}_{2}\right)$ (i.e., any one of the four alternative conditions presented), then $\mathbf{G}$ is also compact:

$$
\left(\mathbf{C}_{1}\right)+\left(\mathbf{C}_{2}\right) \Longrightarrow\left(H_{1}\right)+(4.28) .
$$


Proof. See [15] for details. We note here that only $\left(\mathbf{C}_{1}\right)($ ii) is used to give $\left(\mathbf{H}_{1}\right)(\mathrm{i})$ and that $\left(\mathbf{C}_{1}\right)(\mathrm{i})$,(iii) give a solution of (3.15) initially in $\boldsymbol{\mathcal { V }}:=$ $L^{\bar{p}}([0, T] \rightarrow \mathcal{Y})$ from which one obtains $g:=f(\cdot, x(\cdot)) \in \mathcal{V}$ by Krasnoselskii's Theorem (cf., e.g., [2]). One then has $x \in \mathscr{X}$ from $x=\mathbf{L} g+\mathbf{L} w$ and $\left(\mathbf{H}_{1}\right)$ (i). The four alternative arguments for compactness of $\mathbf{G}$ from $\left(\mathbf{C}_{2}\right)$ use, among them, the Aubin Compactness Theorem [1], the Arzela-Ascoli Theorem, and an argument from [14].

Remark. The conditions above do not apply directly to boundary control (as in Examples 2 and 3) but the arguments in [15] may be easily modifiable to treat this situation. Indeed, the arguments in [15] are fairly standard, using convolution estimates from the form of $\left(\mathbf{C}_{1}\right)(\mathrm{i})$ as applied to the representation (6.51) and the modification simply amounts to the corresponding use of a rather similar representation [3]

$$
z(t)=[\mathbf{L} w](t)=\int_{0}^{t}\left[(-\mathbf{A})^{\vartheta} \mathbf{S}(t-s)\right]\left[(-\mathbf{A})^{1-\vartheta} \Gamma\right] w(s) d s
$$

for the solution of

$$
\dot{z}=\mathbf{A} z, \quad \mathbf{M} z=w:=\mathbf{B}(u) \in \mathbb{W}_{a d} .
$$

Here, A (whose domain formally involves imposition of homogeneous boundary conditions) is the infinitesimal generator of an analytic semigroup $\mathbf{S}$ on $\mathcal{X}$ and $\mathbf{M}$ is the appropriate boundary operator; we have introduced the so-called "Green's operator" $\Gamma: \omega \mapsto v$ by

$$
-\mathbf{A} v=0, \quad \mathbf{M} v=\omega
$$

We then use regularity theory for the problem defining $\Gamma$, the relation of the Sobolev spaces $H^{2 \vartheta}:=\left[L^{2}, H^{1}\right]_{2 \vartheta}$ to the domain of $(-\mathbf{A})^{\vartheta}$ (e.g., [7] or [8]) when $\mathbf{A}$ is a second order elliptic operator, and the estimate (2.9).

We now turn to consideration of the convexity of $\mathcal{Z}_{a d}$ and ask: How restrictive an assumption is this? We have already noted that we may replace $\mathcal{Z}_{\text {ad }}$ by any equivalent set $\mathcal{Z}_{\text {ad }}^{\prime}$ satisfying (3.17) and we shall now see that we may quite reasonably expect to find such a closed convex set $\mathcal{Z}_{a d}^{\prime}$. We will actually work with $\mathbb{W}_{a d}$ (cf. (3.18)) and will find a natural setting for which $\mathbb{W}_{a d} \equiv \mathbb{W}_{a d}^{\prime}:=\overline{c o} \mathbb{W}_{a d}(\overline{c o}=$ closed convex hull in $\mathbb{W})$. We first need a preparatory result, which we "dignify" as a theorem.

Theorem 7. Let $\mathcal{W}$ have the form $L^{p}([0, T] \rightarrow \mathcal{W})[1 \leq p<\infty]$ with $\mathcal{W}$ satisfying the technical hypothesis that ${ }^{6}$

$$
\mathcal{W}_{*}:=L^{\infty}\left([0, T] \rightarrow \mathcal{W}^{*}\right) \quad \text { is dense in } \mathcal{W}^{*} .
$$

\footnotetext{
${ }^{6}$ It is known to be sufficient for this that $\mathcal{W}$ be reflexive or, somewhat more generally, that $\mathcal{W}$ have the Radon-Nikodym Property; cf., e.g., [6].
} 
Suppose a subset $\mathbb{W}_{0} \subset \mathcal{W}$ has ${ }^{7}$ the segment property:

$$
w_{0}, w_{1} \in \mathbb{W}_{0} \Longrightarrow w_{s} \in \mathbb{W}_{0} \quad(s \in \mathcal{S}=\{\text { dense in }[0, T]\})
$$

where, given $w_{0}$ and $w_{1}$, we set

$$
w_{s}(t):=\left\{w_{1}(t) \text { for } 0 \leq t<s ; \quad w_{0}(t) \text { for } s \leq t \leq T\right\} .
$$

Then $\mathbb{W}_{1}$, the sequential weak closure of $\mathbb{W}_{0}$ in $\mathbb{W}$, is just the closed convex hull $\overline{c o} \mathbf{W}_{0}$.

Proof. We set $\Sigma_{\mathcal{S}}:=$ ffinite unions of intervals in $[0, T]$ with endpoints in $\mathcal{S}$ \}; for $\sigma \in \Sigma_{\mathcal{S}}$, let $\chi_{\sigma}$ be its characteristic function and then for $w_{0}, w_{1} \in \mathbb{W}_{0}$ set

$$
w_{\sigma}=\left\{w_{1} \text { on } \sigma ; \quad w_{0} \text { on }[0, T] \backslash \sigma\right\}=\left(1-\chi_{\sigma}\right) w_{0}+\chi_{\sigma} w_{1}
$$

so, e.g., $w_{s}$ - as defined for $(\mathrm{SP})$ - is now $w_{[0, s)}$. Note that repeated application of (SP) shows that each such $w_{\sigma}$ must also be in $\mathbb{W}_{0}$. Note, also, that (SP) for $\mathbb{W}_{0}$ immediately gives (SP) for its sequential weak closure $\mathbb{W}^{\prime}$ : just consider approximating sequences from $\mathbb{W}_{0}$. Since $\overline{c o} \mathbb{W}_{1}=\overline{c o} \mathbb{W}_{0}$, we may assume, with no loss of generality, that $\mathbb{W}_{0}$ is already (sequentially weakly) closed and only show that (SP) then implies its convexity.

Now observe that for any constant $\vartheta \in(0,1)$ there is a sequence $\left\{\sigma_{n}\right\} \subset \Sigma_{\mathcal{S}}$ such that $\left\{\chi_{\sigma}\right\}$ is weak-* convergent to the constant function $\vartheta$ in $L^{\infty}(0, T)$. To see this, just partition $[0, T]$ into $n$ equal subintervals and (noting the assumed density of $\mathcal{S}$ ) choose a sub-subinterval in each with endpoints in $\mathcal{S}$ and having length approximately $\vartheta T / n$; this gives $\sigma_{n}$. The weak-* convergence to $\vartheta$ is then an easy computation.

Given any $w_{0}, w_{1} \in \mathbb{W}_{0}$ and $\vartheta \in(0,1)$, we note, by repeated application of (SP), that each $w_{\sigma}=\left[\chi_{\sigma} w_{1}+\left(1-\chi_{\sigma}\right) w_{0}\right]$ is also in $\mathbb{W}_{0}$. For any $\psi \in \mathbb{W}_{*}$ the $\mathcal{W}$-duality product $\left\langle\psi, w_{1}-w_{0}\right\rangle$ (taken pointwise in $t$ ) is a function in $L^{p}(0, T) \subset$ $L^{1}(0, T)$ so for the $\mathbb{N}$-duality we have

$$
\begin{aligned}
\left\langle\psi, w_{\sigma}\right\rangle & =\int_{0}^{T}\left\langle\psi, \chi_{\sigma} w_{1}+\left(1-\chi_{\sigma}\right) w_{0}\right\rangle d t \\
& =\int_{0}^{T} \chi_{\sigma}\left\langle\psi, w_{1}-w_{0}\right\rangle d t+\left\langle\psi, w_{0}\right\rangle \\
& =\left\langle\chi_{\sigma},\left\langle\psi, w_{1}-w_{0}\right\rangle\right\rangle_{L^{1}(0, T)}+\left\langle\psi, w_{0}\right\rangle \\
& \left.\longrightarrow \vartheta,\left\langle\psi, w_{1}-w_{0}\right\rangle\right\rangle_{L^{1}(0, T)}+\left\langle\psi, w_{0}\right\rangle \\
& =\left\langle\psi, \vartheta w_{1}+(1-\vartheta) w_{0}\right\rangle .
\end{aligned}
$$

Since this holds for each $\psi$ in the dense subset $\mathbb{W}_{*} \subset \mathbb{W}^{*}$, it follows that $w_{\sigma} \rightarrow\left[\vartheta w_{1}+(1-\vartheta) w_{0}\right]$ (weak convergence in $\mathbb{W}$ ) so this is in $\mathbb{N}_{0}$. This, for each such $w_{0}, w_{1}, \vartheta$, is just the desired convexity of $\mathbb{W}_{0}$.

\footnotetext{
${ }^{7}$ Typically, $\mathbb{U}_{a d}$ has the form: $\mathfrak{U}_{a d}=\left\{u \in \mathbb{U}: u(t) \in \mathcal{U}_{a d}(t) \subset \mathcal{U}\right.$ a.e. $\}$ and so satisfies (SP) if, say, $\mathbb{U}:=L^{p}([0, T] \rightarrow \mathcal{U})$. In this case, if $\mathbf{B}$ is defined pointwise in $t$ for (1.2), etc., then (SP) is immediate for $\mathbb{W}_{a d}$.
} 
Corollary. Assume $\left(\mathbf{H}_{1}\right)$. Let $\mathbf{W}, \boldsymbol{W}_{\text {ad }}$ be as in Theorem 7 . Suppose $\mathbf{S}: w \mapsto z: \mathbb{W} \rightarrow \mathcal{Z}$, as in (3.18), is continuous with $\mathbf{E S}: \mathbb{W} \rightarrow \mathcal{X}$ compact and the graph of $\mathbf{G}_{\mathbf{F}} \circ \mathbf{S}$ is closed in $\mathbb{W}_{s w} \times \boldsymbol{V}\left(\cdot \cdot_{\text {sw }}\right.$ indicates the sequential weak topology). Then $\boldsymbol{W}_{a d} \equiv \mathbb{W}_{a d}^{\prime}:=\overline{c o} \mathbb{W}_{a d}$ in the sense of $(3.17)$.

Proof. Since $\mathbb{W}_{a d} \subset \mathbb{W}_{a d}^{\prime}$, we automatically have similar inclusions in (3.17). It is the converse which is effectively a corollary to Theorem 7 .

Fix $\xi \in \overline{\mathcal{K}}_{\mathbf{F}}\left(\mathscr{Z}_{a d}^{\prime}\right)$ where $\mathbb{Z}_{a d}^{\prime}:=\mathbf{S}\left[\overline{c o} \mathbb{W}_{a d}\right]$ so for each (fixed) $\varepsilon>0$ there is some $w=w_{\varepsilon} \in \overline{c o} \mathbb{N} \mathbb{W}_{a d}$ such that $|\xi-\mathbf{T S} w|<\varepsilon$; set $z=\mathbf{S} w$ and $g=\mathbf{G} z$. By Theorem 7 there is then a sequence $w_{k}$ in $\mathbb{N}_{a d}$ with $w_{k} \rightarrow w$. Since $\left\{z_{k}:=\mathbf{S} w_{k}\right\}$ is bounded we may, by $\left(\mathbf{H}_{1}\right)$ (iii), extract a subsequence (still denoted by $w_{k}$ ) with $g_{k}=\mathbf{G} z_{k} \rightarrow \hat{g}$ in $\mathcal{V}$. The closed graph requirement then ensures that $\hat{g}=g$ so, in particular, $\mathbf{L}_{[T]} g_{k} \rightarrow \mathbf{L}_{[T]} g$ in $\boldsymbol{X}$. Since we also have $\mathbf{E S} w_{k} \rightarrow \mathbf{E S} w$ by the assumed compactness, this gives $\mathbf{T S} w_{k} \rightarrow \mathbf{T S} w$. This shows that for some $w_{k} \in \mathbb{W}_{a d}$ we have $\left|\xi-\mathbf{T S} w_{k}\right|<2 \varepsilon$ and that, for each $\varepsilon>0$, proves that we have $\xi \in \overline{\mathcal{K}}_{\mathbf{F}}\left(\mathbb{Z}_{a d}\right)$. Of course this applies for each $\xi \in \overline{\mathcal{K}}_{\mathbf{F}}\left(\mathcal{Z}_{\text {ad }}^{\prime}\right)$. The arguments for $\overline{\mathcal{K}}_{0}$ and $\overline{\mathcal{K}}_{g}$ are similar but simpler.

Note that, with only a small modification of the hypotheses here, one can show that the closures (say, in $\boldsymbol{X}$ ) of the solution sets for (1.2), (1.4), (1.1) will be the same for $\mathbb{W}_{a d}$ as for its (closed) convex hull $\mathbb{W W}_{a d}^{\prime}$

\section{References}

[1] J. P. Aubin, Un théorème de compacité, CRAS de Paris, 1963, pp. 50425043.

[2] J. P. Aubin and I. Ekeland, Applied Nonlinear Analysis. Wiley-Interscience, New York, 1984.

[3] A. V. Balakrishnan, Applied Functional Analysis. Springer Verlag, New York, Berlin, 1976.

[4] J. Bergh and J. Löfstrom, Interpolation Spaces, an Introduction. SpringerVerlag, Berlin, 1976.

[5] N. Carmichael and M.D. Quinn, Fixed point methods in nonlinear control, in Distributed Parameter Systems, Lecture Notes in Control and Information Sci. 75; F. Kappel, K. Kunisch, and W. Schappacher, eds., SpringerVerlag, New York, Berlin, 1985, pp. 24-51.

[6] J. Diestel and J. J. Uhl, Vector Measures. American Mathematical Society, Providence, RI, 1977.

[7] D. Fujiwara, Concrete characterization of the domains of fractional powers of some elliptic differential operators of the second order, Proc. Japan Acad., 43 (1967), pp. 82-86. 
[8] P. Grisvard, Caractérisation de quelques espaces d'interpolation, Arch. Rat. Mech. Anal., 25 (1967), pp. 40-63.

[9] D. Henry, Geometric Theory of Semilinear Parabolic Equations; Lecture Notes in Math. 840, Springer-Verlag, Berlin, 1981.

[10] E. Michael, Continuous selections I, Ann. Math., 63 (1956), pp. 361-382.

[11] K. Naito, Controllability of semilinear control systems, I, SIAM J. Control Optim., 25 (1987), pp. 715-722.

[12] K. Naito, Approximation and controllability for solutions of semilinear control systems, Control-Theory and Advanced Technology 1 (1985), pp. 165173 .

[13] T. I. Seidman, A one-sided miscellany, in Proc. IFAC 4th Symposium on Distributed Parameter Systems, 1986.

[14] T. I. Seidman, Two compactness lemmas, in Nonlinear Semigroups, Partial Differential Equations, and Attractors; Lecture Notes in Math. 1248, T. Gill and W. Zachary, eds., Springer-Verlag, New York, Berlin, 1987, pp. 162168.

[15] T. I. Seidman, Invariance of the reachable set under nonlinear perturbations, SIAM J. Control Optim., 25 (1987), pp. 1173-1191.

[16] T. I. Seidman, Invariance under nonlinear perturbations for reachable and almost-reachable sets, in Control Problems for Systems Described by Partial Differential Equations and Applications, Lecture Notes Control and Information Sci. 97, I. Lasiecka and R. Triggiani, eds., Springer-Verlag, New York, Berlin, 1987, pp. 336-345.

[17] E. Zuazua, Exact controllability for the semilinear wave equation, in Nonlinear Partial Differential Equations and Their Applications, Collége de France Seminar '87-'88, H. Brezis and J. L. Lions, eds., Pitman, to appear. 